\title{
Entre la parole et l'image : le système mythopoétique marubo
}

\section{Pedro de Niemeyer Cesarino}

Traducteur : Pierre Déléage

\section{OpenEdition \\ Journals}

Édition électronique

URL : http://journals.openedition.org/jsa/11739

DOI : 10.4000/jsa.11739

ISSN : 1957-7842

Éditeur

Société des américanistes

\section{Édition imprimée}

Date de publication : 5 octobre 2011

Pagination : 223-257

ISSN : 0037-9174

Référence électronique

Pedro de Niemeyer Cesarino, "Entre la parole et l'image : le système mythopoétique marubo », Journal de la société des américanistes [En ligne], 97-1 | 2011, mis en ligne le 10 décembre 2014, consulté le 20 avril 2019. URL : http://journals.openedition.org/jsa/11739; DOI : 10.4000/jsa.11739 


\title{
ENTRE LA PAROLE ET L'IMAGE : LE SYSTÈME MYTHOPOÉTIQUE MARUBO
}

\author{
Pedro de NIEMEYER CESARINO *
}

Les arts de la parole des Marubo (peuple pano du bassin du Javari, Amazonas, Brésil) entretiennent des relations intrinsèques avec des productions iconographiques et certains dispositifs rituels qui permettent d'identifier les conditions d'une pensée visuelle. L'étude des formules poétiques fait apparaître un schème structural préexistant transposable en signes graphiques et en configurations chorégraphiques. En actualisant un système de formules virtuel, les chamanes orientent trois de leurs arts de la parole vers des finalités spécifiques. Les récits mythiques chantés (saiti), les chants thérapeutiques (shõki) et les chants d'esprits (iniki) s'articulent les uns avec les autres au moyen de formules poétiques prédéterminées, issues d'un champ sociocosmique personnifié. Les relations de transformation et de composition de ces schèmes préexistants forment un savoir mythopoétique de spécialistes, caractérisé par des processus de transmission et d'acquisition qui résultent de la conformation excentrée des cosmologies amérindiennes. [Mots-clés : chamanisme, arts de la parole, iconographie, Marubo.]

Entre o verbal e o visual : o sistema mitopoiético marubo. As artes verbais dos Marubo (falantes de pano da bacia do Vale do Javari, Amazonas, Brasil) possuem vínculos intrínsecos com produções iconográficas e com certas configurações rituais, capazes de apontar para as condições de um pensamento visual. $\mathrm{O}$ estudo das fórmulas poéticas revela um esquema estrutural prévio transponível para signos gráficos e para configurações coreográficas. Atualizando um sistema formulaico virtual, os xamãs movimentam três dos modos das artes verbais de acordo com fins específicos. As narrativas míticas cantadas (saiti), os cantos de cura (shõki) e os cantos dos espíritos (iniki) se articulam em usos determinados das fórmulas poéticas, provenientes do campo sociocósmico personificado. As relações de transformação e composição dos esquemas prévios constituem um conhecimento mitopoético especializado, marcado por processos de transmissão e aquisição que remontam ao caráter exocentrado das cosmologias ameríndias. [Palavras chaves : xamanismo, artes verbais, iconografia, Marubo.]

Between the verbal and the visual: Marubo mythopoetic system. The verbal arts of the Marubo (a Panoan-speaking people from Vale do Javari, Amazonas, Brazil) are

* Professeur de l'université fédérale de São Paulo (UNIFESP), à Guarulhos, Estrada do caminho velho 333, Bairro pimentas, Guarulhos, São Paulo, Brasil [pedroncesarino@uol.com.br].

Journal de la Société des Américanistes, 2011, 97-1, pp. 223-257. C Société des Américanistes. 
systematically connected with iconographic productions and with certain ritual configurations, which seem to indicate the conditions of a visual thought. The study of poetic formulas reveals a previous structural scheme that can be transposed to graphic signs and choreographic configurations. The shamans instantiate a virtual formulaic system by manipulating three of the verbal art modes according to specific ends. Chanted mythical narratives (saiti), curing chants (shóki) and spirit chants (iniki) are articulated in certain uses of the poetic formulae, related to the personified socio-cosmic field. The transformation and composition relationships of the previous schemes constitute a specialized mythological and poetic knowledge, characterized by transmission and acquisition processes that are connected to the exo-centered Amerindian cosmologies. [Key words : shamanism, verbal arts, iconography, Marubo.]

Dans un article consacré à la pensée de Lévi-Strauss, Patrice Maniglier (2008, p. 39) remarque avec justesse que, pour comprendre un événement narratif, il est nécessaire d'avoir accès à son « système mythologique virtuel». Si un tel système, qui « permet de savoir sur quelles différences particulières joue le narrateur », reste hors de portée, la narration n'apparaît guère que comme un « discours en langue étrangère ». Il s'agit là d'une description parfaite de ce que ressent l'auditeur lors des longues séances chamaniques des Marubo, assis sur les bancs parallèles kenã, l'espace rituel masculin situé à l'entrée des malocas (shovo). Gorgés de jus de tabac (rome) et d'ayahuasca (oni), les chamanes y associent une connaissance approfondie des récits mythiques à une agentivité rituelle et à un dispositif d'apprentissage. Ils actualisent, pour ainsi dire, un système virtuel par l'usage particulier de chants thérapeutiques, d'exégèses, de spéculations et d'autres registres sémiotiques, tous issus de processus de transmission stables ${ }^{1}$.

L'étude de ce système virtuel est fondamentale, non seulement pour l'anthropologue qui tente de décoder un savoir cosmologique en grande partie élaboré dans le discours poétique, mais aussi et surtout pour les chamanes eux-mêmes et leurs apprentis. La transmission, dialogique et rituelle, de ce savoir se déroule pour l'essentiel au cours de l'apprentissage des chants shõki, chants de guérison, chants-pensées ou même chants-souffles dans une traduction plus radicale, et des chants-mythes saiti, longues narrations mythiques chantées et versifiées. Ces deux genres discursifs font partie de ce que l'on nomme " paroles pensées », chinã vana, une catégorie définie par un usage spécial du langage qui associe métaphores, énonciations polyphoniques et parallélismes. Cet article ${ }^{2}$ se propose d'étudier les articulations intersémiotiques qu'entretiennent ces arts de la parole avec les configurations rituelles et les schèmes graphiques dans lesquels ils s'inscrivent.

\section{LES ARTS DE LA PAROLE}

Quelle est la relation entre les chants-mythes saiti et les shõki ? Comment le savoir mythologique virtuel peut-il s'actualiser en agentivité rituelle ? Du point de 
vue formel, les deux systèmes appartiennent à un ensemble plus large dans lequel des blocs de formules peuvent être séquencés et emboîtés dans des armatures distinctes. Nous verrons ainsi qu'un ensemble de formules ayant trait, par exemple, au mythe d'émergence des pécaris (yawa), peut être enchâssé dans un chant de chasse destiné à attirer ces animaux à proximité d'un village. Ou encore qu'un chamane souhaitant neutraliser les agressions d'un esprit (yochĩ) peut intégrer dans son chant shõki des vers décrivant la formation et les habitudes de l'entité en question ${ }^{3}$.

Ces mêmes vers peuvent aussi avoir été transmis au chamane-chanteur par un chamane romeya; dans ce cas, les vers appartenaient à un chant-citation iniki. Les romeya, qui appartiennent au second type de chamanes ou de chamanisme des Marubo, correspondant au chamanisme vertical défini par Hugh-Jones (1994) ${ }^{4}$, répètent ce qu'ils entendent des paroles et des chants lointains des esprits yovevo et yochivo. La personne marubo est conçue comme une maloca (shovo) : l'intérieur du corps reproduit la structure spatiale de la maison. Dans cet espace intérieur, les doubles (vaká) des chamanes romeya entretiennent des relations sociales analogues à celles que les Marubo établissent entre eux dans l'espace extérieur, c'est-à-dire dans les malocas où s'assoit l'auditoire des séances chamaniques. Lorsque le double du romeya sort de son corps-maloca pour explorer les régions du cosmos, un esprit ou le double d'un parent décédé prend sa place, et c'est ce dernier qui chante l'iniki. Dans l'exemple suivant, Esprit Coati-Ara (Kana Chichi), provenant de son territoire localisé dans le Monde Arboré (Tama Shavá) ${ }^{5}$, chante à travers le corps-maloca de Cherõpapa. Le chant est composé de termes de la langue des anciens et de formules métaphoriques ${ }^{6}$ dont le sens littéral est traduit dans la troisième colonne (ici comme dans les autres traductions de cet article $^{7}$ ) :

$1 \quad$ kana tawa peiki pesotanairisho e nĩkãkamẽi

« epavo mã epavo

5 ẽ yoe rakati

metsaai venawẽ!! »

txo iki vanamai

nokẽ awe shavovo

Kana Vo aĩvo

10 yove mãsẽ keneya

vevo sanápakemãi

nori rivi ionã

vevo ikãwãki

ẽ oĩpaoa de la feuille de roseau-ara

de là-haut

j'écoutai

« pères, mes pères

venez ici

réjouir ma maloca! »

ainsi parle le cadet

et nos sœurs

la jeune Kana Vo

arrive les mains chargées

de la calebasse-esprit ornée

tandis que nous chantons ${ }^{10}$

y a-t-il eu des changements

depuis le temps /de ma maloca-ara ${ }^{8}$

/de l'intérieur

(11) ${ }^{9}$

(10) 


\begin{tabular}{|c|c|c|c|}
\hline 15 & wetsa aya ravãra? & de ma présence en ce lieu? & \\
\hline & ẽ anõ akara & c'est ainsi que je pense & \\
\hline & e e e yonãke & j’ai fini de parler & \\
\hline & ato pari kanisho & ceux qui autrefois naquirent & \\
\hline & shata ronõ tekepa & morceaux de serpent-léger & /les bancs parallèles ${ }^{11}$ \\
\hline 20 & pati ivevakĩsho & les morceaux assis & /les bancs entiers ils occupaient \\
\hline & vana matsaknãnãvo & conversant entre eux & \\
\hline & ẽ nĩkãpaoa & c'est ainsi que j'entendis & \\
\hline & awesaira & mais pourquoi maintenant & \\
\hline & vanama setesho & restez-vous assis silencieux & \\
\hline 25 & ea nĩkãivo? & m'écoutant seulement? & \\
\hline & Kape Topa aĩvo & Topa, la Femme Caïman & \\
\hline & shokô nane nĩkãi & ne comprit pas qu'elle pouvait & $(28)$ \\
\hline & nĩkã ichnárao & prendre le génipa-pelé & (27) \\
\hline & shokô paka kashkeno & elle aiguisa un couteau & \\
\hline 30 & awẽ ana revo & la pointe de la langue & \\
\hline & te avarãsho & la langue elle tira & \\
\hline & ari ashtekãi & elle se la coupa & \\
\hline & vana ama rakai & et devint muette & \\
\hline & iki kavi ara & comme vous autres & \\
\hline 35 & e e e yonãke & j'ai fini de parler & \\
\hline
\end{tabular}

De manière ironique, Esprit-Coati emprunte un ensemble de formules du chant-mythe de Roka afin de provoquer ses auditeurs qui s'endorment sur les bancs de la maloca. Dans le mythe, l'ancêtre Roka décide de fuir la terre afin que ses parents ne soient pas brûlés : il part pour le Ciel-Pelé ${ }^{12}$ et emmène avec lui son épouse, des fruits de pupunha (Bactris gasipaes) et de génipa ainsi qu'une torche enflammée. Il appelle ensuite ses autres parents et leur demande de l'accompagner, mais ceux-ci refusent. Il offre alors le génipa à ceux qui resteront sur terre afin qu'ils puissent régénérer leur corps à l'aide de sa teinture. Topa, une FemmeCaïman, comprend mal les paroles de Roka et se mutile : elle croyait qu'il avait ordonné à chacun de se couper la langue - d'où l'origine de l'une des caractéristiques saillantes des caïmans jusqu'à ce jour. L'extrait est placé ici dans un but ironique et didactique : le mode narratif est déplacé ou enchâssé à l'intérieur du mode reportatif. De plus, le locuteur a commencé son chant par une polyphonie (trait général des chants chamaniques amérindiens ${ }^{13}$ ) : il a cité ce que le double du chamane (nommé « cadet ») lui avait dit, à savoir qu'il était invité pour réjouir son corps-maloca (vers 4 à 7). Accompagné par sa sœur, l'esprit arrive sur terre et est déçu par ce qu'il y trouve : les hommes ne s'y consacrent plus avec autant de persévérance qu'autrefois au chamanisme et à l'écoute de ses chants.

Courts et mélodieux, les iniki contrastent avec le rythme régulier des longs chants shõki et saiti. Lorsqu'un esprit chante à travers le corps-maison du romeya, 
l'auditoire doit rester attentif au message de son discours. Les chamaneschanteurs, pour la plupart des shõikiya, peuvent par exemple écouter un ensemble de formules versifiées où Esprit-Anaconda parle de sa formation ou de son surgissement, du lieu dans lequel il vit, de son nom ou de ses habitudes. Après avoir mémorisé ces informations versifiées, le chamane-chanteur pourra les utiliser dans d'autres circonstances afin de composer un chant shõki susceptible d'apaiser les maux engendrés par cet esprit en particulier. Les trois arts de la parole étudiés dans cet article, les chants-mythes saiti, les souffles-chants shõki et les paroles des esprits iniki, ne sont pas exactement des genres classifiés dans une typologie rigide. Tous utilisent un ensemble virtuel de formules poétiques indépendant de ses actualisations en modes narratifs (saiti), agentifs (shõki) ou reportatifs (iniki). En fonction d'un événement singulier, l'ensemble virtuel pourra être orienté vers un de ces trois modes qui sont, de surcroît, susceptibles de s'enchâsser les uns à l'intérieur des autres. Ainsi, un chant iniki pourra contenir un extrait de shõki qui, à son tour, pourra contenir un extrait de chant-mythe. Alternativement, un chant-mythe peut intégrer un souffle-chant ou un chant iniki dans une de ses séquences. Il ne s'agit pas, dans chacun de ces cas, d'analogies ou d'allusions ; il s'agit de modifications de l'orientation du système de formules.

\begin{tabular}{|l|l|l|}
\hline \multicolumn{3}{|c|}{ Modes des arts de la parole } \\
\hline chants-mythes & saiti & mode narratif \\
\hline chants d'esprits & iniki & mode reportatif \\
\hline souffles-chants & shõki & mode agentif \\
\hline
\end{tabular}

Au cours d'une conversation à propos des chants shõki et de la multiplicité des esprits du cosmos marubo, Armando Cherõpapa entonna le fragment de chant suivant. Ce faisant, il transmettait succinctement des ensembles de formules correspondant à la formation des doubles des anacondas, sous la forme d'un fragment susceptible d'être intégré au sein d'un long chant shõki.

1 atõ mane roeyai

roeyai oneki

vei tama niáki

atõ vake reraa

5

rakápakemaĩnõ

vei tama tekeyai

tekeyai oneki

vei waka shakĩni

aya onepakeki

$10 \quad$ vei waka shakini

aya shokoakesho

vei tama tekeki

atõ aya onea

\author{
leurs haches de métal \\ les haches ils cachèrent \\ et arbre-mort \\ leurs fils abattirent \\ et une fois tombé \\ le tronc d'arbre-mort, \\ le tronc ils cachèrent ${ }^{14}$ \\ dans fleuve-mort \\ ils l'y cachèrent \\ dans fleuve-mort \\ ils l'y placèrent \\ le tronc d'arbre-mort \\ ils le laissèrent caché
}




$\begin{aligned} & \text { Ene Mai tsakamash } \\ & 15 \quad \text { ene meã tsakamash } \\ & \text { rakãnavo atõ ash } \\ & \text { vei shõpa peiki } \\ & \text { votĩki irinõ } \\ & \text { mai rakákãisho } \\ & \text { rakãnivo yochĩra- } \\ & \text { yochĩvoro eakiki } \\ & {[\ldots] }\end{aligned}$

\author{
dans Terre-Fleuve bouillonnante \\ dans la rivière bouillonnante \\ ils l'y laissèrent ${ }^{15}$ \\ et feuille de stramoine-mort \\ la feuille ils mélangèrent \\ et sur terre la placèrent \\ les esprits allongés - \\ c'est moi l'esprit, dit-on ${ }^{16}$
}

Comme le montre l'emploi de la particule - $k i$ à la fin du fragment, Cherõpapa est bien en train de citer les paroles de l'esprit ou du double ${ }^{17}$ de l'anaconda. La formule de clôture de ce bloc (« les esprits allongés/c'est moi l'esprit, dit-on ») indique que l'histoire de la formation de l'esprit, issue d'un chant-mythe saiti, est focalisée ou orientée vers un mode agentif. Sachant comment l'esprit s'est formé, ce que celui-ci a lui-même relaté dans la séquence d'un mythe, Cherõpapa pourra faire usage du chant pour soigner une série de maux dont le spectre est probablement à l'origine. L'histoire explique comment, il y a très longtemps, les corps des grands anacondas se formèrent: des esprits démiurges (les tierces personnes auxquelles le chant fait allusion) placèrent les "troncs d'arbre-mort » dans le «Fleuve-Mort » et c'est ainsi qu'apparurent les corps des grands anacondas. Ce processus de formation aurait pu être raconté sous la forme d'une simple narration (yoã vana) ou d'un chant dénué d'intention agentive. En effet, il est issu du Kanã Mari mai vana saiti (« Kanã Mari, la formation de la terre »), un mythe qui raconte comment la terre a été formée par les esprits démiurges Kanã Mari. Cependant, dans le fragment cité, les modes reportatif et narratif ont été orientés vers un mode agentif.

Les chants shõki sont ainsi en grande partie associés à des récits mythiques précis dont l'ensemble de formules poétiques est réorienté, en fonction des circonstances, vers la mythopraxis. L'inventaire de l'intégralité des chants-mythes correspondant à chacune des sections des chants shõki est pratiquement impossible à effectuer. Les Marubo connaissent des centaines de longs chants-mythes saiti comprenant les histoires des Wenía, les cycles de surgissement du cosmos, des fleuves, de la configuration actuelle de la Terre, des étrangers, des ancêtres, des animaux et des plantes ainsi qu'une kyrielle d'intrigues se déroulant dans l'espace-temps du surgissement (weníanamã). Dans la mesure où la transcription d'un seul chant s'étend sur un minimum de quelques dizaines de pages, il est difficile de rendre compte de manière exhaustive d'une telle tradition : la récitation correcte d'un seul saiti peut durer plusieurs nuits. Aujourd'hui, la plupart des chamanes ne les connaissent plus dans leur intégralité. Les souffles-chants, quant à eux, sont destinés à guérir certaines maladies ou à retrouver les doubles perdus (vaká rapakea). Ils permettent aussi de prendre en charge toute une série de 
problèmes d'ordre sociocosmique : congédier les esprits (yochĩ) qui tourmentent les vivants, rendre la chasse heureuse, modifier le climat et l'atmosphère, activer des remèdes et d'autres vecteurs de "pensée-vie " (chinã) comme le mingau de banane ${ }^{18}$, l'ayahuasca et le jus de tabac ou, encore, agir par la pensée sur divers collectifs tels que les étrangers, les esprits ou les ennemis.

\section{Formules ET CLASSIFICATEURS}

Revenons aux propos de Cherõpapa concernant les anacondas et les autres doubles susceptibles d'être contrôlés par les chants shõki. L'appréhension rituelle complète des doubles agressifs des anacondas (responsables du redouté « mal de l'anaconda », vẽchã ichná, qui entraîne le dépérissement de sa victime) s'effectue en plusieurs étapes. Il est nécessaire de savoir chanter (1) la formation de ses corps (awẽ kaya) puis (2) celle de ses doubles qui implique la connaissance de leur lieu de surgissement (awẽ wenía), des trajectoires de leurs migrations (awẽ vai), du lieu où ils se sont installés (awẽ tsaoa), de leur nom et de leurs habitudes, en particulier alimentaires. Ainsi, comme les autres entités du cosmos, l'anaconda est composé de son extension corporelle ou de sa carcasse (awẽ kaya, awẽ shaká) et de ses doubles (awẽ vaká) qui, dans ce contexte, sont des personnes (yora) douées de sociabilité.

À chaque action des esprits ancestraux Kanã Mari correspond une formule qui modifie l'axe paradigmatique du chant : plutôt que de mentionner la manière dont le tronc d'un «arbre-mort» (vei tama teke) a été caché, comme dans l'exemple cité, le chant aurait pu choisir d'évoquer les manipulations de troncs d'açai, de samaúma ou de tiges de stramoine qui tous donnèrent naissance à différents corps d'anaconda. La pensée mythopoétique des Marubo est un système complexe de substitution et de séquençage dont nous allons donner quelques exemples ${ }^{19}$. Le terme « mort», apposé à tous ces noms de fragments, est, nous l'avons dit, un classificateur précisant le champ référentiel du chant : la Terre, le Monde de la Terre-Mort (Vei Mai Shavaya) autrefois transformé par les esprits Kanã Mari. Pour chaque référence à la cartographie mythique (ainsi que pour d'autres variations non spatiales), la pensée marubo utilise un classificateur spécifique. Par exemple, les entités appartenant au Monde du Ciel-Sang (Imi Nai Shavaya) apparaissent toujours accompagnées du classificateur «sang »: imi awá, tapir-sang, imi vẽcha, anaconda-sang, imi shono, samaúma-sang, imi nawavo, peuple-sang, etc. Afin de penser et de contrôler les esprits issus de cette strate céleste, le chant combine les informations des axes syntagmatique et paradigmatique de telle sorte que l'appréhension des variations intensives de ce collectif d'esprits soit rendue possible ${ }^{20}$. Cherõpapa utilise un procédé similaire pour penser la nourriture (awẽ yanika) des doubles des anacondas: 
vei shõpa eneki

ene yaniawai

rakãnivo yochĩra

vei oni eneki

5

ene yaniawai

vei oni sanini

vei sanikãisho

rakãnivo yochĩra

vei shõpa eneki

10 ene yaniawai

vei shõpa weki

we txiwamashõta

rakãnivo yochĩra -

yochivoro eaki

15 vei kapi eneki

ene yaniawai

vei kapi sanĩni

vei sanĩkãisho

rakãnĩvo yochĩra -

20

yochĩvoro eakiki

vei rome eneki

ene yaniawai

vei rome sanĩni

vei sanĩkãisho

25

rakãnivo yochĩra

vei rome paẽnõ

ari merakãisho

Ene Mai chinãki

chinãtari awai

30 rakãnivo yochĩraki

[...] infusion de stramoine-mort,

l'infusion ${ }^{21}$ ils viennent boire

les esprits allongés

infusion de liane-mort

l'infusion ils viennent boire

torpeur de liane ${ }^{22}$-mort

de torpeur ils s'enivrent

les esprits allongés

infusion de stramoine-mort

l'infusion ils viennent boire

et vent de stramoine-mort

le vent ils adjoignent ${ }^{23}$

les esprits allongés -

c'est moi l'esprit

infusion de mata-pasto-mort

l'infusion ils viennent boire

torpeur de mata-pasto-mort

de torpeur ils s'enivrent

les esprits allongés -

c'est moi l'esprit, dit-on

infusion de tabac-mort

l'infusion ils viennent boire

torpeur de tabac-mort

de torpeur ils s'enivrent

les esprits allongés

avec la force de tabac-mort

en eux, ils se transforment

et vers Terre-Eau

vers la terre ils se dirigent

les esprits allongés, dit-on

Les doubles des anacondas (également nommés « spectres », yochĩ, puisque tout spectre est le double, vaká, d'un corps) consomment des substances équivalentes à celles qu'ingèrent les chamanes marubo : stramoine, mata-pasto (Senna alata), ayahuasca (Banisteriopsis caapi) ou tabac (Nicotiana tabacum). Ces substances possèdent des vertus transformationnelles comme la «force-tabac » (rome pae), les « vents » (we) et les « torpeurs » (sanĩ). Une fois transformés par ces puissances, les doubles des anacondas partent vivre dans leur monde subaquatique (Ene Mai Shavaya) comme l'indique la formule Ene Mai chinãki / chinãtari awai, « et vers Terre-Eau / vers la terre ils se dirigent ». 
Mes conversations avec Cherõpapa au sujet de la multiplicité inépuisable de doubles et d'esprits de la cosmologie marubo contiennent un autre exemple de transposition du mode mythique au mode agentif. Il me racontait l'histoire du surgissement des doubles du sapajou (chinõ vaká) : ce sont les doubles de ce singe lubrique qui, en se frottant aux personnes, les rendent dévoyées, libidineuses et potentiellement incestueuses. L'origine de ces comportements, typiques des jeunes et des prostitué(e)s des villes, remonte aux temps mythiques : pour réformer ces conduites inadéquates par une action rituelle efficace, cette origine doit être retrouvée. Encore une fois, le bloc formé par le long chant shõki utilisé pour contrôler les harcèlements des doubles lubriques du sapajou enchâsse une séquence du chant-mythe Wenía (« le surgissement des ancêtres »). Après avoir effectué un long trajet, de leur lieu de surgissement à l'aval, « au pied du ciel » (naí taeri, une région identifiée aujourd'hui à Manaus), jusqu'aux sources et au soleil couchant (vari katõ), les anciens chefs Waka Panã et Waka Võko se retrouvèrent devant le Pont-Caïman, élément que l'on retrouve dans d'autres mythologies pano. Les chefs et les chamanes, les savants, traversèrent en premier ce pont monstrueux dont les côtes étaient constellées de plantes comestibles. Une fois de l'autre côté, ils appelèrent leurs parents dévoyés (tanasmarasĩ) qui n'avaient pas écouté leurs paroles et qui, restés en arrière, jouaient avec leur pénis. Lorsque ces derniers atteignirent le milieu du pont, les chefs le tranchèrent et les « dévoyés » se noyèrent dans les eaux du grand fleuve Noa.

$\begin{array}{lll}1 & \text { Rovõ Vake Nawavo } & \text { Fils du Peuple Cassique } \\ \text { Waka Võko inisho } & \text { Waka Võko accompagné } \\ \text { Waka Panã akavo } & \text { de Waka Panã } \\ \text { Rovo Kape Tapãne } & \text { au Pont-Caïman-Cassique }{ }^{24} \\ \text { vake onemashõta } & \text { leurs fils ils conduisirent } \\ \text { Rovo Kape Tapãki } & \text { et du Pont-Caïman-Cassique } \\ \text { atõ osho roenõ } & \text { avec leurs haches-blanc } \\ \text { tetxoiki reraa } & \text { le cou ils tranchèrent } \\ \text { Kape Tewã Tapãki } & \text { et Pont-Caïman-Cassique } \\ \text { asoake kawãki } & \text { fut alors renversé } \\ \text { atõ vake naoa } & \text { et les fils se noyèrent } \\ \text { atõ mão vakaki } & \text { leurs doubles solitaires } \\ \text { ene kewã inisho } & \text { les lames-eau accompagnées } \\ \text { shawã make shetaya } & \text { des piranhas-ara mordantes } \\ \text { atõ vake yashaa } & \text { les fils déchiquetèrent }{ }^{25} \\ \text { atõ mão vakaki } & \text { leurs doubles solitaires }{ }^{26} \\ \text { rapakekãi } & \text { se détachèrent } \\ \text { noa tae irinõ } & \text { et au pied du fleuve } \\ \text { ivai inivoita } & \text { tous se dirigèrent } \\ \text { noa voro wetsanõ } & \text { vers terre-fleuve } \\ \text { ea yochĩ veonõ } & \text { pour que moi spectre je m’y assois }\end{array}$




$\begin{array}{ll}\begin{array}{l}\text { iki awákãi } \\ \text { veõnivo yochĩraki }\end{array} & \text { ainsi ils demeurèrent } \\ {[\ldots]} & \text { les esprits allongés, dit-il }\end{array}$

Après la chute du pont, les descendants du Peuple-Cassique (Iskonawavo) ${ }^{28}$ sont mis en pièces par les lames-eau et les piranhas-ara. Puis ils se dirigent vers l'aval du fleuve et s'installent au sommet d'une des collines de la « Terre-Fleuve », c'est-à-dire dans les demeures du grand fleuve Noa, identifiées aux villes des Blancs. C'est depuis cet emplacement que les doubles continuent jusqu'à ce jour à harceler les vivants et à les rendre lubriques. L'histoire est ici orientée vers une finalité cosmopratique (vers 21-23) : dans une composition complète, elle aurait été combinée avec une série de blocs propres aux chants shõki. Cette actualisation de contenus mythiques est inséparable d'une série de substitutions et de variations de schèmes de formules. En d'autres termes, la multitude d'entités potentiellement agressives qui peuplent le monde («tout est source de mal», disait un chamane) rend nécessaire un exercice permanent d'actualisation des processus de formation des temps mythiques ${ }^{29}$, exercice qui ne devient possible que par les substitutions et les séquençages de stock de formules.

Un autre chamane-chanteur, Antonio Brasil Tekãpapa, me raconta une autre version du processus de formation des anacondas. En guise d'explication, il ne composa pas de chant, mais énuméra une suite de formules poétiques : il s'agissait, une fois encore, de simples amorces de chants. Aux temps du surgissement, des morceaux de liane d'ayahuasca furent cette fois utilisés pour donner naissance aux grands anacondas (vẽcha tokopama). À nouveau, les classificateurs accompagnant les morceaux - ces signes de transformation - furent transposés aux sujets-anacondas : tous étaient classés comme " passereau » ${ }^{30}$, " ornement », « froid » ou « mort », au même titre que l'élément originel, les tiges de l'ayahuasca.

\begin{tabular}{|l|l|l|l|l|l|}
\hline \multicolumn{1}{|c|}{$\mathbf{A}$} & \multicolumn{1}{c|}{$\mathbf{B}$} & \multicolumn{1}{c|}{$\mathbf{C}$} & \multicolumn{1}{c|}{$\mathbf{D}$} & \multicolumn{1}{c|}{$\mathbf{E}$} & \multicolumn{1}{c|}{$\mathbf{F}$} \\
\hline vari & oni & tekepashõki & vari & vẽcha & rasini \\
\hline shane & oni & tekepashõki & shane & vẽcha & rasiñi \\
\hline matsi & oni & tekepashõki & matsi & vẽcha & rasinin \\
\hline vei & oni & tekepashõki & vei & vẽcha & rasini $i$ \\
\hline kana & oni & tekepashõki & kana & vẽcha & rasini $i$ \\
\hline rane & oni & tekepashõki & rane & vẽcha & rasinni \\
\hline
\end{tabular}

\begin{tabular}{|l|l|l|l|l|l|}
\hline \multicolumn{1}{|c|}{ C } & \multicolumn{1}{c|}{ B } & \multicolumn{1}{c|}{ A } & \multicolumn{1}{c|}{ F } & \multicolumn{1}{c|}{ E } & \multicolumn{1}{c|}{ D } \\
\hline fait de morceaux d' & ayahuasca & soleil & collectif & anaconda & soleil \\
\hline fait de morceaux d' & ayahuasca & passereau & collectif & anaconda & passereau \\
\hline fait de morceaux d' & ayahuasca & froid & collectif & anaconda & froid \\
\hline fait de morceaux d' & ayahuasca & mort & collectif & anaconda & mort \\
\hline fait de morceaux d' & ayahuasca & ara & collectif & anaconda & ara \\
\hline fait de morceaux d' & ayahuasca & ornement & collectif & anaconda & ornement \\
\hline
\end{tabular}


Ces formules sont composées d'un élément transformationnel (teke-, " morceau d'ayahuasca »), d'une information grammaticale (-pashõ $k i$, « fait de ») et de classificateurs (soleil, passereau, froid, mort, ara, ornement). Tous ces composants sont ensuite regroupés dans une structure paralléliste. Chaque singularité est marquée par un classificateur qui indique le segment social auquel ses doubles appartiennent. Ce système de classification rituel est ouvert : il peut incorporer de nouveaux termes si l'entité l'exige. Dans l'exemple ci-dessus, Tekãpapa énuméra toute la série de variations des personnes et des collectifs issus du processus de transformation. En général, elle demeure implicite au cours de l'apprentissage puisque les classifications de l'élément transformationnel (la tige d'ayahuasca dans l'exemple) correspondent à celles de la singularité émergente (son corps et ses doubles).

Les doubles des anacondas sont des personnes : elles assument une position d'énonciateurs des chants et entretiennent des relations sociales explicitées par les classificateurs. Ainsi, lorsqu'un double de l'anaconda-passereau (shane vẽcha) chante par le biais d'un romeya, les membres de l'auditoire sauront définir les relations de parenté que ces derniers entretiennent avec lui, une fois révélé son nom ou sa section (Peuple-Passereau, Shanenawavo). Dans le cosmos personnifié des Marubo, les relations de parenté s'étendent indéfiniment, bien au-delà du socius visible, ce qui rend possible l'enlèvement du double d'une personne vivante par un anaconda. Le système de classification régissant les variations parallélistes des formules poétiques constitue ainsi une des façons d'affronter la prolifération sociocosmique de parenté et ses risques potentiels. Les classificateurs ne peuvent toutefois pas être réduits à une transposition de qualités ou à la simple application d'un modèle sociologique aux collectifs du cosmos. Un anaconda-froid, matsi vẽcha, n'est pas un anaconda congelé, mais une entité appartenant à la classe « froid ». Ce qui est généralement explicité de manière laconique : " c'est son nom », "c'est ainsi que parlaient les ancêtres ». Tout se passe comme si les classificateurs formaient un système autonome : c'est pour cela que certains éléments du " parler-penser » ne proviennent pas directement de la mythologie, bien qu'ils partagent avec elle un même schème de formules. Les formules articulent poétiquement les transformations structurales qui transitent ainsi d'un art de la parole à un autre.

Il importe de souligner que le terme "classificateur" se réfère ici à un phénomène de variation positionnelle et non à la problématique de la prototypicalité (présente dans la logique de termes qui ne seront pas abordés ici, tels que -ewa, kaya, koĩ, entre autres) ${ }^{31}$. Valenzuela (2000, p. 24) établit, pour le Shipibo-Conibo, une distinction entre composés (compounds) morphologiques et sémantiques :

kashin mentsis, "griffes de chauve-souris ", est un composé morphologique car il contient une combinaison de deux racines nominales, kashi et mentsis, qui interagissent sémantiquement au sein d'un unique processus métaphorique. Kashin mentsis est une 
espèce de liane qui possède, entre autres caractéristiques, des épines aiguisées, pensées par les Shipibo-Conibo en relation avec les griffes des chauves-souris. Par contraste, oxe bina (oxe "lune » + bina " guêpe ») est un composé sémantique qui se réfère à une espèce de guêpe [...] dont on dit que le nid ressemble à la lune.

Dans le second cas, deux processus sémantiques sont présents : l'un, métaphorique, compare la forme du nid à celle de la lune tandis que l'autre, métonymique, identifie la « guêpe » au « nid ». La langue marubo connaît de nombreux composés de ce genre, comme yotá sheta (littéralement "dent de mygale », désignant métaphoriquement le treillis de paille qui sert de rideau à la porte principale de la maloca) ou shawã ina (littéralement « queue d'ara », une métaphore désignant spécifiquement les foyers). Comme pour la langue shipibo, la composition est également utilisée pour différencier les espèces (Valenzuela 1998). Le système chamanique de classification semble prendre appui sur ce fondement pour en présenter une inflexion originale car, s'il ne présuppose pas de relation génitive (comme dans la « dent de mygale »), il permet de former des noms composés (" tabac-brume » et non « tabac de la brume ») liés à des variations positionnelles.

Mais revenons aux répertoires de formules et, en particulier, aux exemples des ordinateurs, de l'essence, des montres, des lampes torches et d'autres marchandises du monde des Blancs. Le système virtuel permet de les penser de la même manière que les anacondas ou les pécaris. "Tout est pensée de chamane» (katsese kẽchĩtxo chinãrivi) disait-on souvent, signifiant ainsi que les artefacts des Blancs ne constituaient pas d'authentiques nouveautés : le système virtuel se définit avant tout par son ouverture à la multiplicité et à sa traduction dans des schèmes préétablis. Voici une suite s'appliquant aux objets manufacturés :

\begin{tabular}{|c|c|c|c|c|c|c|c|}
\hline $\mathbf{A}$ & B & $\mathbf{C}$ & $\mathbf{C}$ & B & $\mathbf{A}$ & & \\
\hline$t x i$ & kamã & shaõshki & fait d'os de & jaguar & feu & amonauti & lampes torches \\
\hline txi & kamã & verõshki & fait d'yeux de & jaguar & feu & vari oĩti & montres \\
\hline sĩki & kamã & voshká & fait de tête de & jaguar & vertige & vari oĩti & ordinateurs, télévisions \\
\hline sĩki & kamã & sheni & fait de graisse de & jaguar & vertige & veishti & miroirs \\
\hline vopi & kamã & sheni & fait de graisse de & jaguar & mort & veishti & miroirs \\
\hline shetxi & kamã & sheni & fait de graisse de & jaguar & tension & veishti & miroirs \\
\hline tsoka & kamã & sheni & fait de graisse de & jaguar & frisson & veishti & miroirs \\
\hline
\end{tabular}

Ces éléments provenant des Blancs sont « pensés » au moyen d'images verbales. Penser (chinã) signifie aussi faire (shovia) quelque chose : « cela fut fait par la pensée du chamane » (kẽchĩtxo chinã shovirivi), était-il souvent expliqué. Cette forme de pensée agentive et poétique mobilise ainsi des éléments déterminés et les articule dans des formules verbales afin de mieux les comprendre. Certaines des formules se référant au surgissement des choses (comme txi kamã verõshki, " fait d'yeux de jaguar-feux ») peuvent ensuite être simplifiées (en supprimant la 
séquence de morphèmes -shki, «fait de ») devenant alors des noms métaphoriques : txi kamã vero, " yeux de jaguar-feux », devient dans la langue ordinaire (veyô vana) vari oĩti, " chose pour voir le soleil », c'est-à-dire « montre ». Le terme " métaphore » a été traduit, ou reconceptualisé, de diverses manières par les jeunes professeurs marubo. L'une d'elles convient parfaitement au contexte : la métaphore est le nom d'une chose (awẽ ane) utilisé pour penser ou pour « déclencher son surgissement » (awe anõ shovima) ${ }^{32}$.

Dans le tableau présenté ci-dessus, les classificateurs employés pour distinguer les artefacts des Blancs sont les mêmes que ceux utilisés dans plusieurs autres chants shõki destinés à apaiser les maladies. "Vertige », " mort », « tension », « frisson », en plus d'être des effets provoqués par de tels éléments (mais aussi par d'autres esprits agressifs), constituent aussi un ensemble de classificateurs grâce auxquels le chanteur identifie le monde ou la position particulière et vers lesquels se dirigera le chant, à savoir le «monde-mort». La pensée marubo peut ainsi articuler noms et classificateurs afin de prendre en compte l'ensemble des existants. Même s'il emploie des moyens limités (des éléments sélectionnés pour penser un ensemble déterminé), le système s'applique potentiellement à un domaine infini (en intégrant tout ensemble potentiellement pensable). Les relations de voisinage entre les ensembles d'éléments permettent au système de penser des entités et des phénomènes aussi divers que les anacondas, les pécaris, les tapirs, mais aussi les escargots, les hannetons, les guêpes, le tabac, l'ayahuasca, les ennemis étrangers, les phénomènes météorologiques, les objets manufacturés ou les doubles des morts.

\section{RITUEL ET INTERSÉMIOTICITÉ}

Lorsque survient la mort, les corps cessent d'abriter les doubles résidant dans leur maloca intérieure et quelques-uns parmi les plus intelligents doivent emprunter le dangereux Chemin-Mort (Vei Vai), qui relie le Monde de la Terre-Mort au Monde du Ciel-Mort. Les doubles y rencontrent une série d'obstacles et de tentations (fruits sucrés, femmes séductrices, jolis ornements à portée de main, trous remplis de sang, épines gigantesques, etc.) si terribles que fréquemment ils rebroussent chemin et reviennent sur terre. Épouvantés par l'aspect dantesque de ce trajet eschatologique, les doubles demeurent ici-bas et tourmentent leurs parents. Ne parvenant pas à rejoindre leur destination finale, ils ne réalisent pas qu'ils sont morts et leur proximité excessive avec les vivants provoque toutes sortes de maladies.

Afin de neutraliser l'action de ces esprits de morts désorientés, les chamanes entonnent une série de chants semblables aux shõki. Ils ont pour effet soit d'effrayer les spectres en mobilisant l'armée d'esprits auxiliaires shoma, une multitude d'entités bardées de lances, de feux, de boucliers et de jaguars, soit de 
les balayer de la surface de la terre en invoquant de grandes tempêtes. Ces processus se déroulent au cours des rituels kenã txitõna et shavá saika qui permettent aussi bien d'expulser les doubles des morts et de les conduire à leur destination finale que de modifier les conditions atmosphériques. La première cérémonie fournit aussi l'occasion d'apprendre le chant du Chemin-Mort, Vei Vai Yoiya, qui prépare les vivants aux périls de leur traversée post mortem. Comme lors de la transmission des chants saiti, le chamane chante des séquences particulières de formules qui sont ensuite répétées par l'ensemble des participants. Les auditeurs répètent ces paroles et élaborent une chorégraphie imitant le dangereux cheminement.

L'événement s'inscrit dans un dispositif intersémiotique : la chorégraphie reproduit, sur terre et dans le corps des danseurs, le parallélisme du chant. Les sections successives du chant du Chemin-Mort, délimitées par des blocs parallèles qui se distinguent les uns des autres par des substitutions et des concaténations, sont reproduites par les figures de danse. C'est ainsi que le parallélisme, technique de mémorisation et de visualisation ${ }^{33}$, devient pourvu d'une efficacité rituelle. Tout en leur communiquant un contenu narratif agencé par un schème virtuel de formules, les chamanes permettent aux participants du rituel de visualiser la scène décrite par le chant-panorama et de l'incorporer au cours d'une expérience musicale et chorégraphique. En plus de ces registres discursifs, graphiques et cinétiques, l'intersémioticité met en jeu le registre musical, puisque la structure des cellules du chant est également agencée par des variations et des réitérations ${ }^{34}$.

En 2006, au cours de la fête Kenã Txitõna dirigée par le romeya Robson Venãpa, les participants, des chamanes guérisseurs expérimentés pour la plupart, élaborèrent une chorégraphie reproduisant la sinuosité du Chemin-Mort (Figures 1 à 6). De jour comme de nuit, ils entraient et sortaient des malocas dispersées tout du long de la place du village Alegria (Haut Ituí) et chantaient les vers du chant du Chemin-Mort et du chant pour l'Orientation des doubles (Vaká Yonoa), une version amazonienne du Livre des Morts tibétains. Harcelée par les doubles de parents récemment décédés qui avaient échoué dans leur traversée du Chemin-Mort, la communauté Alegria organisa une fête de quatre jours au cours de laquelle l'action rituelle s'accomplit en même temps que l'apprentissage d'arts de la parole plus narratifs. Voici un fragment de cette version du chant pour l'orientation des doubles des morts ${ }^{35}$ : 

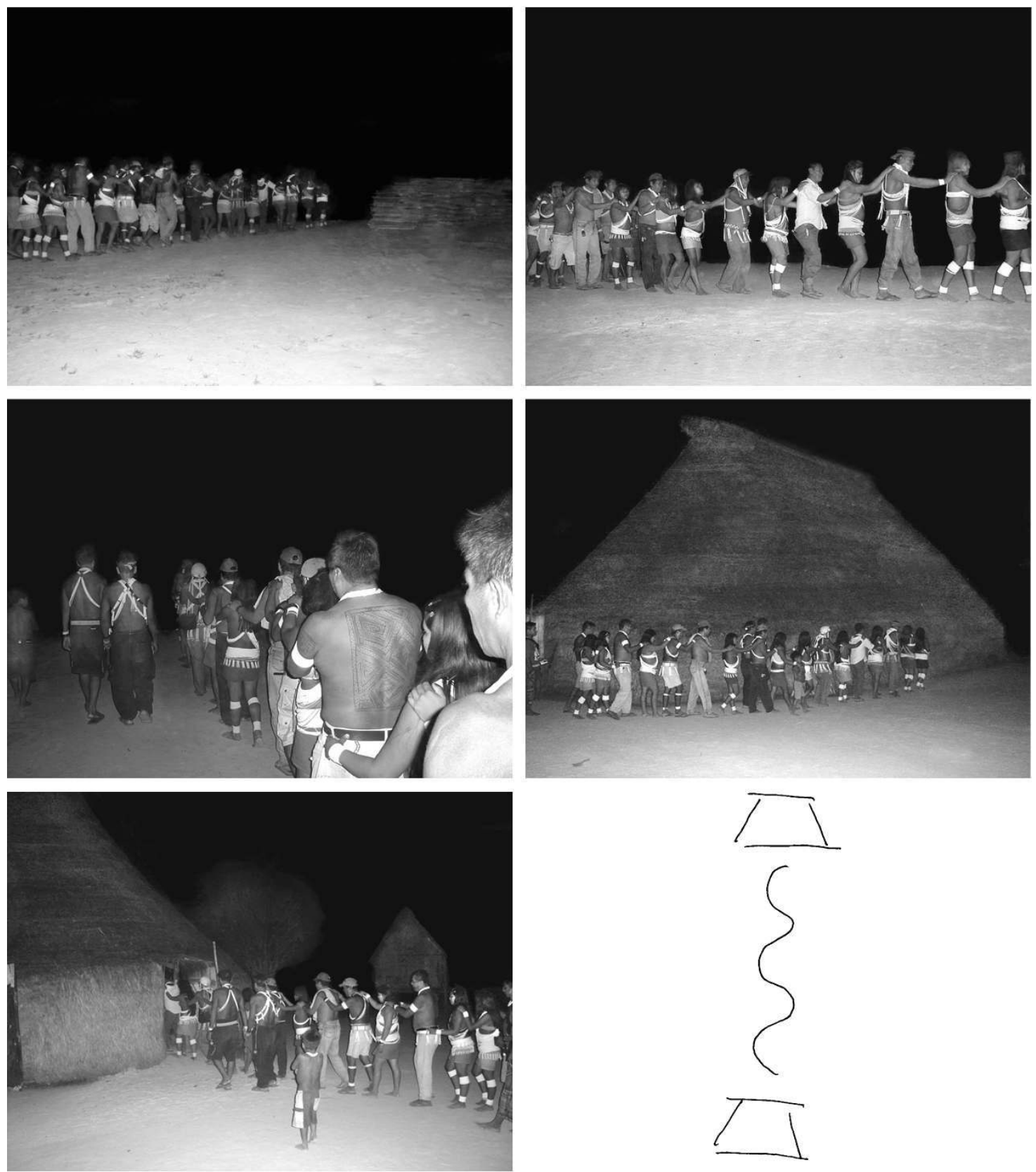

FIG. 6 - Chorégraphies de la fête Kenã Txitõna, village Alegria, Haut Itui (clichés de Cesarino 2006). 
[...]

\section{Ouverture du chemin}

mĩ sheni txovo

anõ iti vairao

80 Vei Mai Shavaya shavá tapiakesho mĩ nete sanãti sanã koũ inisho neri veso oanimai

85 Vei Vai Shavaya shavá tapiakesho vei rono ipapa kayainakãi kai koũ katãwẽ

90 vei rono kashõno kayainakãi neri veso oanimai kai koĩ katãwẽ nea mai shavaya

95 shavá noitivisi mĩ eneakõti vei rono kashõno kayainakãi

\section{Pont-Mort}

Vei Waka yapokash

100 vei awá shao rakãini otivo vei awá shaono kaya ina ikirao kai koĩ katãwẽ !

105 Vei Vai shavaya kayainakãi kai kõ̃ katãwẽ ! neri veso oanimai mi nete sanati

110 sanã koĩ inisho Vei Vai Shavaya kayaina iriwẽ ! Vei Vai Shavaya

115 kayaina ikirao

\section{Tortue-Mort}

vei shoni pei votĩ iki irinõ Vei Vai matoke vei tama shaká de tes anciens

le chemin menant

ici sur Terre-Mort

est ouvert

avec les yeux lucides

regarde bien

et ne te retourne pas

ouvert, Chemin-Mort

il est ouvert

par la queue de serpent-mort

/par le chemin

entreprends l'ascension

pars immédiatement!

par la colonne de serpent-mort /par le chemin

entreprends l'ascension

et ne te retourne pas

pars immédiatement!

le monde de cette terre

le monde chéri

tu vas l'abandonner

par la colonne de serpent-mort

entreprends l'ascension

à travers Fleuve-Mort

os de tapir-mort

autrefois fut placé

par l'os de tapir-mort

entreprends l'ascension

pars immédiatement!

par Chemin-Mort

entreprends l'ascension

pars immédiatement!

ne te retourne pas

avec les yeux lucides

regarde bien

par Chemin-Mort

pars immédiatement !

par Chemin-Mort

entreprends l'ascension

sur la feuille de taioba-mort

sur la feuille courbée

de la colline du Chemin-Mort

de l'écorce d'arbre-mort

/cuir ${ }^{38}$ 
120 awẽ shasho atõ vei shawe ativo Vei Vai kepomash vei shawe ativo mia vake tawẽmisi

125 «ẽ vero tsakánõ!» mĩ kamẽ iamai

\section{Singe-Mort}

vei tama voro masotaná irinõ vei iso ativo

130 tsaoini otivo " vei yõká ativo ẽ yanianõnã!» mĩ kamẽ iamai mia veiyamisi

\section{Sang-Mort}

135 vei rono imi vei vai teamash vei rono shavã nasotaná irino vei mai mîkini

140 tsaoini otivo vei imi ativo machitain vãin vei moshô wenõ

145 teki inakãi

Vei Vai shavaya shavá tapiakesho kayainakãirao neri veso oanimai

150 kai koĩ katãwẽ! mĩ otxi txovo mĩ txitxo txovo atõ merainii kai koĩ katãwẽ!

\section{Fruits-Mort}

155 « vei shãta ativo ẽ yanianõnã!! »

mĩ kamẽ iamai

« vei shõpa ativo

ẽ yaniañ̃nã!» est faite sa carapace

tortue-mort autrefois

ferma Chemin-Mort ${ }^{39}$

tortue-mort autrefois

sa cheville voulut couper

« tortue je veux regarder!»

ne pense pas ainsi

sur la souche d'arbre-mort

/colline-mort ${ }^{40}$

là-haut

singe-mort était ${ }^{41}$

autrefois assis

« goyaves-mort ils firent

pour me rassasier ! »

ne pense pas ainsi

tu pourrais mourir

sang de serpent-mort ${ }^{42}$

ferme chemin-mort

morceau de serpent-mort

là, au fond

de trou-mort

autrefois fut placé 43

par-dessus sang-mort

bondis immédiatement

avec le vent d'arbre-mort ${ }^{44}$

léger, entreprends l'ascension

ouvert, Chemin-Mort

il est ouvert

entreprends l'ascension

ne te retourne pas

pars immédiatement!

tous tes frères 45

toutes tes sœurs

tu vas retrouver

pars immédiatement !

« fruit de la passion-mort ils firent

pour me rassasier!»

ne pense pas ainsi

« papaye-mort ils firent

pour me rassasier!» 


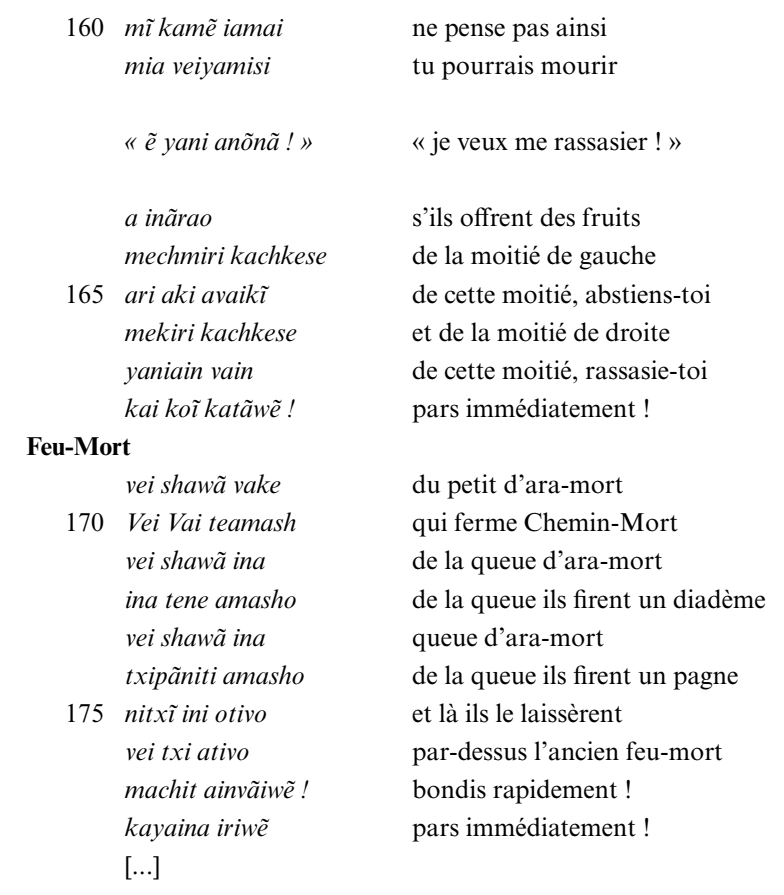

C'est en dialoguant avec le double du mort récalcitrant que le chamane le conduit le long du dangereux chemin. Au début, le mort souhaite rester avec ses parents et il semble ne pas bien connaître les obstacles. C'est pour cela qu'il a besoin d'être orienté : probablement n'avait-il pas appris, au cours de sa vie, les paroles qui lui auraient permis de franchir ces obstacles. Chaque étape du trajet est divisée en blocs parallèles plus ou moins longs, indiqués par des sous-titres dans les extraits cités. Pendant la danse, les blocs chantés par le meneur sont répétés par les danseurs afin qu'ils mémorisent l'ordre fixe des séquences (Severi 2007). Dans le schème stable des formules, les substitutions se référant aux diverses étapes du chemin apparaissent progressivement, ponctuant un trajet continu par des pauses discontinues. La formule métaphorique pour le pontanaconda glissant, vei tama voro, « tronc d'arbre-mort $»{ }^{46}$, sera suivie de formules conservant toutes, à gauche, le classificateur «mort», par exemple vei rono imi, « sang de serpent-mort».

Plusieurs enchaînements exposent également les processus de formation ou de composition des entités. Ainsi, les vers 171-176 décrivent la genèse du «feumort », qui fut formé grâce à un diadème fait des plumes d'un petit d'ara ${ }^{47}$. On trouve d'autres enchaînements de ce genre dans les versions intégrales du chant et dans d'autres arts de la parole marubo. L'apprenti du chamane (et, dans le cas du Chemin-Mort, tout le monde) doit connaître parfaitement ces enchaînements qui 
racontent la formation ou l'origine (shovia, wenía) des choses et des entités. C'est ainsi que l'on apprend l'art de manipuler les singularités. Celles-ci apparaissent scindées entre doubles et corps : c'est par la connaissance exacte des formules poétiques et de leur système de substitutions que le chanteur devient capable de manœuvrer le flux personnifié de cosmos lié à une telle scission. Le savoir mnémotechnique impliqué par l'usage de ces structures parallélistes et de ces compositions de formules ne doit pas être réduit à son aspect rhétorique ou à sa fonction de stabilisation de l'information, il constitue également une esthétique de l'intensité réitérative aux usages cosmopratiques.

\section{LES UNITÉS VISUELLES}

Pour que le chanteur-penseur devienne capable d'utiliser ses chants, il doit avoir accès à un schème virtuel qui s'actualise dans divers registres sémiotiques. On ne s'étonnera donc pas que ces structures narratives puissent être transposées en signes graphiques, comme le montre une étude de quelques dessins réalisés à ma demande. Ainsi, le chamane-chanteur Tekãpapa accepta de dessiner le Chemin-Mort sous la forme reproduite Figure 7.

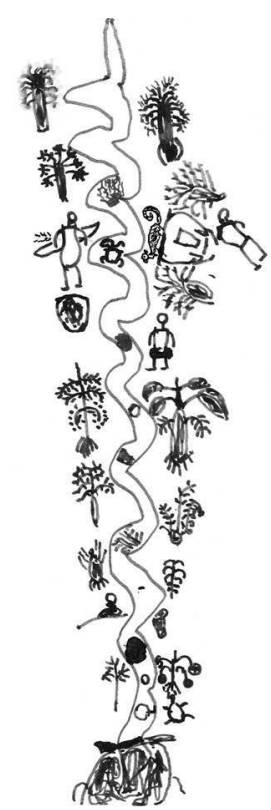

FIG. 7 - Le Chemin-Mort (Vei Vai), d'après un dessin d'Antonio Brasil Tekãpapa (2004), crayons de couleurs sur papier $210 \times 297 \mathrm{~mm}$. 
La composition montre le Chemin-Mort qui relie la terre au monde du Ciel-Mort. En bas, on voit la maloca des doubles des yeux, où vivent les parents du défunt. Tekãpapa, également l'auteur de la version du chant pour orienter les morts, est analphabète. Il est incapable d'écrire son nom en entier et n'avait jamais produit de tels dessins avant ma demande. Or sa transposition des séquences narratives sur le papier apparaît rigoureusement schématique. Dans la section centrale du dessin, on peut observer une maloca entourée de trois figures humanoïdes : le singe au sommet de la maloca correspond aux vers 127-130 du chant (singe-mort ou singe atèle); la tache (rouge dans l'original) à gauche d'une figure humanoïde, au milieu du chemin, correspond aux vers 135-140 (sang-mort) ; la première figure (en rouge dans l'original) de la moitié du haut, au milieu du chemin se réfère, quant à elle, aux vers 169-170 (feu-mort), et ainsi de suite.

Il est probable que certains schèmes graphiques (composés au minimum d'une ligne, sinueuse ou non, figurant un trajet et des traits parallèles représentant la superposition des strates célestes) étaient autrefois dessinés sur le sable par les chamanes marubo. Je n'ai vu qu'une seule fois Cherõpapa esquisser quelques traits parallèles sur le sol en contrepoint de ses explications cosmologiques. La précision avec laquelle Tekãpapa reproduit les éléments du chemin, sans même mentionner la dextérité de son tracé, n'est surpassée que par celle d'Armando Cherõpapa. Ce dernier réalisa un grand dessin du même chemin sur cinq feuilles de papier, collées les unes aux autres. Puisque son double avait vu le chemin alors qu'il était " suspendu dans le vent » (wẽs a ronorivi), Cherõpapa était en mesure de le décrire avec plus de précision que Tekãpapa qui n'en avait qu'entendu parler. Le schème préalable, acquis par l'expérience immédiate ou par le biais du discours des autres, rendit possible la reproduction des étapes du chemin sur le papier : toutes correspondent aux formules poétiques du chant.

Il devient dès lors possible de présenter de manière plus systématique les moments et les unités minimales qui structurent à la fois les chants et les autres registres sémiotiques. Nous avons vu comment les doubles des anacondas se transformèrent après l'ingestion de leurs substances et se dirigèrent vers le monde subaquatique où ils vivent encore. Dans l'eschatologie, les morts partent aussi de leur maloca d'origine, située sur Terre, parcourent un trajet déterminé sur le Chemin-Mort, puis s'installent dans la maloca des doubles des yeux. Le schème général comprend donc trois moments : surgissement, trajet et installation.

Les dessins ne sont pas le produit d'une libre inspiration : traduisant des formules poétiques en signes graphiques, ils transposent un schème général préexistant en faisant usage d'un ensemble précis d'unités visuelles. Le cercle (1) permet de désigner soit le lieu de surgissement d'un collectif ou du protagoniste d'un récit, soit les mondes, les demeures et les collines, respectivement shavá et matô, parfois représentés sous forme d'une maloca (2), shovo. Les traits (3), wichá, sont utilisés pour figurer les trajets de surgissement et de migration (wenía vai), que ceux-ci soient terrestres, fluviaux ou célestes. Les humanoïdes (4), yora, 
représentent des personnes et/ou des collectifs, parfois accompagnés de diacritiques indiquant leur appartenance à une configuration sociale donnée (emblèmes et ornements corporels, peintures faciales, couleurs, par exemple). Ces signes graphiques, dans la mesure où ils transposent des moments narratifs, correspondent à des formules particulières (voir, en annexe, les tableaux 1 à 4 qui comportent également d'autres types de signes). Manipulant ces correspondances, Armando Cherõpapa transposa sous forme de dessin-cosmogramme quelques vers du chant du Surgissement des Chamanes, Kẽchĩtxo Wenía (Figure 8$)^{48}$.

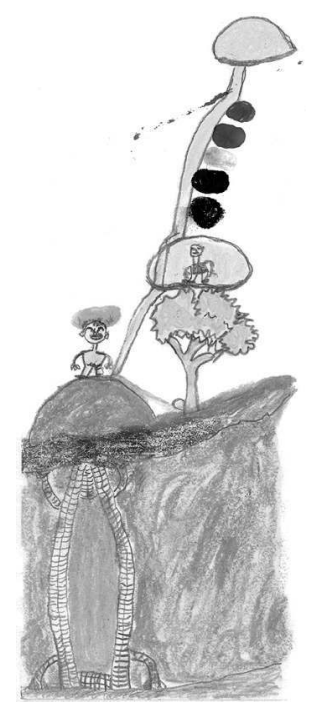

FIG. 8 - Le Chemin de la Tortue Blanche, d'après un dessin d'Armando Cherõpapa Txano (2006), feutres et crayons de couleurs sur papier $210 \times 297 \mathrm{~mm}$.

On y observe un chamane-chanteur, un "Pajé-Blanc», surgir du «nectar d'Arbre-Blanc » (tama osho nãkõsh wenía) pour ensuite s'installer dans son monde. Le lieu de surgissement est signalé par un petit cercle situé au niveau de la souche de l'arbre. Un premier trait indique le chemin parcouru jusqu'à la colline (une métaphore visuelle pour la maloca ou le village où vivent les humanoïdes). Cette séquence initiale, appartenant au chant-mythe du surgissement des chamanes, est la traduction de l'enchaînement de formules narratives suivant : « du nectar d'arbre-esprit / du nectar répandu / l'esprit surgit / et dans collineesprit, / là ils vont vivre » (yove tama nãko / nãko osõatõsho / yove shovivãini lyove mai matoke / nioi kaoi). Dans la section inférieure, Cherõpapa a dessiné le Pont-Caïman, précisant ainsi que la scène se déroule à l'aval du fleuve, sur l'autre rive du grand fleuve Noa, le « lieu du surgissement » (weníanamã), et non dans 
la région des sources où vivent aujourd'hui les Marubo. Après s'être installé dans la colline, le double du chamane se dirige vers le monde de la Cime des Arbres (Tama Shavá) comme le montrent les signes superposés humanoïde et cercle, dessinés au sommet de l'arbre. Il empruntera ensuite le chemin de la Tortue Blanche (Shawe Osho Vai) qui longe les diverses strates célestes (représentées par des cercles coloriés ${ }^{49}$ ) et atteindra la terre du Tabac Blanc (Rome Osho Mai), une « terre-esprit » (yove mai), la destination post mortem réservée aux chamanes et à leurs épouses.

Ce schème visuel n'est pas seulement un cosmogramme, il est aussi caractérisé par une logique diachronique représentant un cycle vital. La temporalité marubo est pensée en fonction d'un axe aval/amont : les événements qui ont eu lieu «par là » (okiri, en direction de l'embouchure) sont les plus anciens et se rapportent, en général, aux " temps du surgissement »; ceux qui se sont déroulés "par ici » (nekiri, en direction des sources) appartiennent au passé récent. Le cadre diachronique global du dessin-narration-cosmogramme correspond à un cycle vital particulier, celui du chamane, qui surgit du « nectar d'arbre-esprit », trouve son monde et entreprend le trajet qui le conduira à sa destination post mortem. Le terme « arbre » est une métaphore désignant "l'homme» (esprit), tandis que « nectar » signifie « semence ». Une fois décodé, ce discours métaphorique explique donc que le chamane fut engendré par le Peuple-Esprit de l'arbre, à partir de son sang et de sa semence. Le dessin transpose le sens métaphorique du discours qui reste la plupart du temps inaccessible aux Marubo novices ou non initiés. Comme dans les chants de la première partie de cet article, un même schème virtuel s'actualise dans diverses configurations fondées sur les moments-clés (surgissement, trajet et installation), et dans ses articulations à des signes graphiques et à des ensembles de formules.

Il convient de distinguer au moins deux répertoires graphiques marubo : le premier se rapporte aux motifs kene et le second aux dessins ici étudiés. Les kene, motifs d'apparence géométrique dont l'étude précise nous mènerait trop loin, sont réalisés par les femmes dans des situations et sur des supports variés (peintures et ornements corporels, céramique, paraphernalia rituels, etc.). Les dessins qui nous intéressent ici ne forment pas, quant à eux, un répertoire ancien et ils ne sont exécutés que par les hommes, en particulier par les chamanes les plus vieux. Les premiers semblent se prêter à un usage agentif : la transformation ou l'altération des supports auxquels ils s'appliquent (par exemple, le corps) ${ }^{50}$. Les dessins des chamanes correspondent, pour leur part, à des transpositions visuelles précises de formules verbales et sont accompagnés par une pragmatique plus limitée issue de la relation à l'anthropologue et du projet d'élaboration d'un livre. Ces dessins sont composés de signes visuels qui constituent un système de différences et de relations (voir, en annexe, les tableaux 1 à 4) ${ }^{51}$. Ils ne sont pas des figurations ou des illustrations et ne peuvent pas être qualifiés de "dessins spontanés » (Déléage 2007, p. 117). Ils se restreignent à la figuration de listes 
et de séquences narratives et on y observe soit une « association conventionnelle entre un signe et un nom », soit une « homologie entre un ordre des signes et un ordre du discours » (Déléage 2011). C'est ainsi, par exemple, qu'une variante du signe humanoïde pourra être associée au nom d'un habitant particulier du cosmos marubo, ou qu'une séquence de signes composés pourra correspondre à une formule spécifique et à sa réitération paralléliste (voir, en annexe, les tableaux 1 à 4).

Malgré l'absence d'une tradition locale ancienne et/ou stabilisée, cette iconographie partage plusieurs traits communs avec d'autres systèmes pictographiques amérindiens. Tout se passe comme s'il existait une matrice de pensée antérieure à sa transposition schématique sur un support ou dans un média particulier. À partir de cette matrice, il devient possible de contrôler une série de modes d'expression qui se retrouvent ainsi structurellement liés. Chants, dessins, mélodies et danses articulent, au moyen de procédures sémiotiques distinctes, les trois moments fondamentaux (surgissement, trajet et installation) dans de grands ensembles organisés par la réitération, la juxtaposition et la séquenciation ${ }^{52}$. C'est justement la présence de cette matrice ou de ce schème préexistant qui permet la création soudaine et apparemment mystérieuse d'un corpus iconographique systématique et rigoureux, dont les caractéristiques générales apparaissent similaires à celles de répertoires amérindiens stables (tels que ceux des Navajo, des Kuna ou des Ojibwa, par exemple). Mais comment une telle matrice peut-elle être transmise ? Il convient maintenant de proposer quelques éléments de réponse à cette question.

\section{LE CHAMP SOCIOCOSMIQUE}

Au cours d'un long apprentissage, le chamane-chanteur acquiert petit à petit la maîtrise des schèmes de formules que lui transmettent ses maîtres. Ces derniers ont, eux aussi, appris ces vers en compagnie d'un chamane-chanteur ou, du moins, ils ont écouté ce qu'un chamane-esprit disait par le truchement d'un chamane. Les esprits sont aussi des chamanes, plus savants encore que ceux des Marubo. La composition spéciale de la personne chez les Marubo, où le corps reproduit à l'échelle interne la maloca et ses configurations sociales (Cesarino 2008), permet aux chamanes de s'emboîter les uns dans les autres, interférant ainsi à tous moments sur le processus de transmission de ce savoir. Dans une cérémonie collective d'initiation, un chamane des esprits-oiseaux, les espritsgrive (yove mawa), rendit visite au corps-maloca de Cherõpapa, participant ainsi à l'enseignement délivré. Une autre fois, un chamane kapanawa vint dans Robson Venãpa afin de transmettre ses longs chants. Le « double du cœur » (chinã nató) de Robson Venãpa, nommé Isko Osho, avait autrefois entretenu des relations avec ce chamane kapanawa, dont il avait appris plusieurs chants et danses ${ }^{53}$. 
« Nous ne pouvons achever ces paroles, des paroles comme celles-ci n'ont pas de fin » me disaient-ils. La prolifération des formules poétiques tend à l'infini et ce, pour au moins deux raisons : leurs variations syntagmatiques et paradigmatiques sont indéfinies et les sources d'énonciation et d'élaboration de leurs messages sont également virtuellement inépuisables. Il n'existe pas de centre (autorité du chef-sacerdote, subjectivité créatrice, intériorité responsable de projections animistes, etc.) à partir duquel puisse être énoncé le savoir chamanique ${ }^{54}$. Le schème est décentré ou excentré. Ce qui rappelle les systèmes de transformations des Mythologiques qui inspirèrent à Viveiros de Castro cette réflexion : «Le créateur n'est pas sujet ou substance mais pure relation : intermédiaire, véhicule, nœud, médiateur, transmetteur $\gg{ }^{55}$. Les émetteurs appartiennent potentiellement à ce que Viveiros de Castro (2002) décrit comme un «champ infini de virtualité sociale » : c'est à partir de ce champ, et à son encontre, que fonctionne la mythopoïesis marubo. Poiêin, poiêsis : les esprits démiurges « fabriquèrent » ( shovima) le cosmos à partir d'éléments déjà épars dans les paysages primordiaux (morceaux d'animaux, lianes d'ayahuasca, entre autres). De nos jours, les chanteurs actualisent ce processus de transformation en employant les variations de formules verbivisuelles qui composent leur chinã, leur pensée ou leur principe vital, leur capacité de « fabriquer » virtuellement des singularités et de les manipuler par le biais de paroles transformatives.

Le système mythopoétique marubo ne s'appuie pas seulement sur l'expérience visionnaire, comme chez d'autres peuples pano : il s'articule autour de problèmes d'action cosmopratique, de traduction et de citation. La consommation de l'ayahuasca (sans additif tel que la chacrona, Psychotria viridis) permet de contracter une alliance avec son esprit, Oni Shãko, "Pousse d'Ayahuasca », c'est-à-dire son « maître » ou son « double » (oni ivo, oni vaká). Situé auprès du chanteur qui ingère le breuvage, ce double va l'aider (takea, "s'accoter ») à chanter, par exemple un long shõki. L'objectif de l'ingestion constante de substances chamaniques, comme l'ayahuasca ou le jus de tabac, est d'obtenir de leurs maîtres et de leurs doubles qu'ils facilitent l'apprentissage et la manipulation des chants. Avec l'aide permanente de ces Autres (maîtres des substances, doubles des parents morts, esprits-oiseaux auxiliaires, etc.), le chamane devient progressivement capable de mémoriser et de manipuler les systèmes de formules. On pourrait dire qu'il est déjà multiple : sa pensée est « liée » (chinã ãtiya) à celle d'interlocuteurs autres qui se détachent du " champ infini de virtualité sociale » et qui s'allient à lui. Le vaste système de concaténations de formules exige un champ tout aussi vaste de relations sociocosmiques : alliés, parents, interlocuteurs, tels sont les protagonistes de cette épistémologie chamanique. Ainsi, les conditions de production du savoir chamanique trouvent leur fondement dans un champ relationnel décentré ${ }^{56}$.

Une Babel complexe dans laquelle surviennent de nombreux esprits, des parents morts et d'autres subjectivités : c'est ainsi que l'on peut décrire une séance 
chamanique marubo typique. La maloca dans laquelle le romeya chante (et enseigne le schème de formules à son auditoire) n'est qu'un point dans l'interminable réseau de relations qui constitue le cosmos marubo. Ce que l'on voit du romeya, c'est sa carcasse (shaká), un genre de transmetteur acoustique (« il est comme un microphone », microfone keská) de dialogues entamés ailleurs, entre son double et ses interlocuteurs autres. Au cours du dialogisme chamanique, les interlocuteurs se détachent d'un arrière-plan virtuel et se mettent en relation grâce à l'idiome conceptuel de la parenté, marqué par le système de classificateurs qui oriente le sens et l'usage des formules poétiques. Une représentation du corps d'un chamane romeya, recueillie par Delvair Montagner, illustre parfaitement ce point (Figure 9).

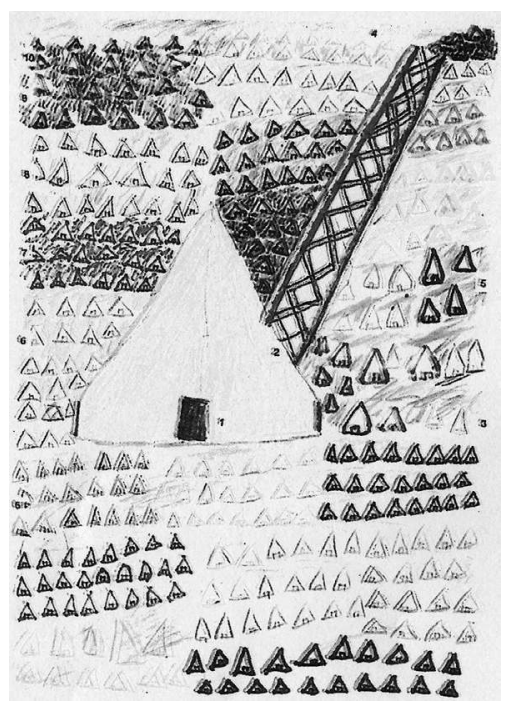

FIG. 9 - Représentation du cosmos et du corps d'un chamane, Firmínio Marubo (Montagner 1996)

La maloca au centre du dessin (un cosmogramme qui reproduit les différentes strates célestes à l'aide de couleurs différentes) est le corps du chamane ou, plutôt, ce que ses doubles internes conçoivent, de leur propre point de vue, comme une maloca. Il ne s'agit pas d'une métaphore (« Le corps est une maloca », "Les Bororo sont des aras », etc.), mais de différences de position et de réflexivité. En suivant un chemin décoré de motifs en zigzag, les doubles internes du romeya ont accès aux $\mathrm{n}$ malocas des habitants du cosmos : les trajets de leurs voyages sont très précisément décrits par les chants iniki, dont les formules poétiques correspondent à des signes visuels particuliers (voir, en annexe, le tableau 3). C'est de cette myriade personnifiée que surgit le savoir, 
c'est-à-dire la maîtrise des formules ou plutôt du complexe mécanisme de variations et de concaténations par lequel elles sont engendrées, condition de possibilité du transit entre les multiples champs référentiels.

Dans ce panorama, quel espace est laissé à l'invention ? À partir d'un schème préexistant, le chanteur recombine des éléments dans le cadre d'une armature que lui et ses pairs connaissent déjà. Ces éléments ne se restreignent pas à un ensemble clos puisque leurs variations sont potentiellement infinies, comme le sont les peuples, les mondes, les personnes et les esprits. Comme pour d'autres peuples amérindiens, l'extériorité est centrale et c'est d'elle que surgiront toujours de nouveaux éléments (noms de personnes, de lieux, de substances, de collectifs, etc.) au cours d'un événement chamanique. C'est précisément là ce qui fonde la réputation d'un chamane: savoir véhiculer, transmettre et rendre possible de telles actualisations d'un champ cosmique inaccessible en son entier, en les mettant au service d'une finalité pratique. La pensée marubo est une pensée du vertige : par l'effort rituel ininterrompu de ses chamanes, par la prolifération stupéfiante de ses schèmes mythopoétiques, elle apparaît préoccupée par ce qui toujours se dérobe et à jamais excède les possibilités des humains vivants, l'infini. *

* Manuscrit reçu en octobre 2009, accepté pour publication en janvier 2011.

\section{Notes}

1. Les références au virtuel et à la multiplicité ont pour origine la cosmologie des Marubo (Cesarino 2008) et se rapportent, d'une manière générale, à la pensée de Deleuze (in Deleuze et Guattari $1991)$ et à son usage par Viveiros de Castro $(2002 ; 2006 ; 2007)$. Cette référence n'est pas une nouveauté dans l'américanisme comme on peut le constater à la lecture de la célèbre « Ouverture » des Mythologiques de Lévi-Strauss (1964). Toutefois le virtuel ne se limite pas ici au seul ordre des transformations structurales : il concerne aussi le champ sociocosmique propre aux Marubo.

2. Cet article présente et approfondit certains des sujets développés dans ma thèse de doctorat (Cesarino 2008). Il a été traduit du portugais par Pierre Déléage. Les étapes successives de cette recherche ont reçu l'appui du CNPq et de la FAPERJ (PRONEX), du CNRS (EREA, Bourse du Legs Lelong), de la Wenner-Gren Foundation et de la FAPESP.

3. J'utilise ici la notion de «formule poétique» au sens de Lord (1981, p. 30) : « un groupe de mots employé régulièrement dans des conditions métriques identiques afin d'exprimer une idée essentielle ». La notion ne doit pas être confondue avec celle de "formule canonique du mythe » de Lévi-Strauss (analysée par Almeida 2008) même s'il existe des affinités entre les deux registres. Tout se passe comme si le système de formules des arts de la parole pouvait, à certains moments, être mis au service des transformations structurales qu'opère la "formule canonique" dans le registre narratif. Dans le cas marubo, les formules poétiques forment des dispositifs mythopoétiques dont la validité ne se restreint pas aux opérations essentiellement narratives repérées par Lévi-Strauss.

4. La distinction entre chamanisme vertical et chamanisme horizontal proposée par Hugh-Jones a fait l'objet d'une réinterprétation à partir de la notion de « chamanisme transversal » par Viveiros de Castro (2008). 
5. Tama est un terme générique utilisé pour désigner les arbres.

6. Les formules poétiques marubo sont assez proches, selon plusieurs aspects (métrique, lexique, grammaire, sémantique), de celles d'autres traditions pano : sharanawa (Déléage 2006), kaxinawá (Guimarães 2002 ; Lagrou 1998), yaminawa (Townsley 1993 ; Naveira 2007) et shipibo-conibo (Tournon 1991). Une analyse comparative plus précise de ces ressemblances reste à effectuer. Une étude approfondie des formules marubo et de leurs régimes poétiques et métaphoriques dépasserait, quant à elle, de très loin les limites de cet article.

7. Les traductions de cet article sont des extraits révisés d'exemples issus de ma thèse de doctorat. Ce travail fut effectué en compagnie de Marubo bilingues et de chamanes-chanteurs à partir de mes propres enquêtes linguistiques et ethnographiques. Les traductions ont été pensées comme des re-créations littéraires, plutôt que comme des versions littérales; elles sont ici adaptées du portugais par Pierre Déléage. Dans l'orthographe marubo, «e » est une voyelle centrale supérieure, «sh » une affriquée alvéopalatale et « ch » une rétroflexe.

8. Au vers 1, ce que nous percevons comme des feuilles de roseau (marquées ici par le classificateur « ara » car l'esprit appartient au Peuple-Ara, Kananawavo), l'esprit le voit comme sa propre maison. D'où la «torsion » du discours qui résulte moins d'une distinction entre un sens objectif (vrai) et un sens dérivé (fictif) que d'un problème de position : ainsi ce qui est dit d'une certaine manière par un locuteur, par exemple un esprit, doit parfois être considéré d'une autre manière par un autre locuteur, par exemple un Marubo vivant.

9. La numérotation indique une inversion de lignes dans la traduction libre.

10. Les esprits ne se déplacent pas seuls dans le cosmos : ils sont toujours accompagnés de leurs parents et de leurs sœurs qui arrivent en dansant, les mains chargées d'une calebasse d'ayahuasca.

11. Shata rono teke (ou, dans d'autres versions, shata rono tosha) signifie, pour les esprits, « bancs parallèles ». La métaphore semble fondée sur la ressemblance entre le corps des serpents et le bois utilisé pour les bancs.

12. «Pelé » (shokô) est également un classificateur marquant les éléments liés au niveau céleste du Monde du Ciel-Pelé (Shokô Naí Shavaya) et aux processus de régénération (comme la desquamation) qui y ont lieu. On reviendra plus loin sur le rôle des classificateurs dans la pensée marubo.

13. Pour une étude comparative, Cesarino (2003) ; pour les chants tupi, Viveiros de Castro (1986) et Oakdale (1996, 2005) ; pour les arts de la parole kuna, Sherzer (1990) et Severi (1996).

14. Depuis toujours, les esprits possèdent des instruments métalliques (et des armes à feu), disent souvent les chamanes. Il s'agit ici d'indiquer comment les esprits déplacèrent in illo tempore la matière première utilisée pour «faire» (shovima) les corps des anacondas. On remarquera la présence du classificateur « mort » qui fait référence non seulement aux entités et aux éléments du « Monde de la Terre-Mort » (Vei Mai Shavaya), c'est-à-dire notre monde, mais aussi aux processus de corruption et de désagrégation, aux maladies et aux phénomènes d'extinction qui le caractérisent (par opposition, par exemple, aux processus de renouvellement typiques du monde et des qualités liées au classificateur « pelé », shokô).

15. «Terre-Fleuve bouillonnante» fait référence au monde (shavá) subaquatique, ses habitants le voyant comme une terre. «Fleuve-Mort» ne désigne pas un fleuve précis de la cartographie marubo, mais un fleuve générique ou exemplaire propre au « Monde-Mort».

16. Les yochĩ, esprits particulièrement agressifs, apparaissent «debout» (niá) ou « allongés » (raká) : ces positions caractérisent différents types d'esprits associés à différents processus de surgissement (et d'agentivité). Les esprits provenant du vomi d'un malade desséché par le soleil, par exemple, apparaissent "allongés " sur le sol, prêts à attaquer les passants. Les esprits des maîtres morts des essarts sont, quant à eux, « debout », à côté de leurs bananeraies, enviant leurs parents. On remarquera également l'usage de la stramoine (Brugmansia sp.) comme substance transformationnelle susceptible de donner forme aux esprits. Les deux matières premières dont se servent les démiurges Kanã Mari sont les troncs d'arbre, pour la création des corps ou des carcasses (shaká) des anacondas, et les feuilles de stramoine, pour la création de leurs doubles ou esprits (vaká, yochĩ), c'est-à-dire de leurs " personnes » (yora). Pour plus de détails, voir Cesarino (2008). 
17. Ce que je nomme " singularité » est une entité composée d'un corps (yora) d'une carcasse ( shaká) ou d'une extension corporelle (kaya) et d'un ou de plusieurs doubles (vaká), humanoïdes ou non. À chaque support corporel correspond un double, relié au corps par des relations de possession et de réflexivité (Cesarino 2008).

18. Le mingau de banane (mani motsá), composé de banane crue ou cuite dissoute dans l'eau, est employé dans le cadre de divers rituels chamaniques des Marubo.

19. On trouvera dans Cesarino (2008) des traductions et des analyses détaillées. Voir aussi Montagner (1996) pour la traduction libre d'un chant shõki.

20. Dans cet article, le choix de traduction des noms composés a été longuement réfléchi. Comme on le verra, il s'est agi de transposer dans la langue cible une dynamique implicite de variation et de classification : c'est ce qu'il faut garder à l'esprit à la lecture de constructions du type « samaúmasang », qui ne se réfère pas à un arbre ensanglanté, ou " papaye-mort », qui n'indique pas un fruit mort, mais une papaye de la terre-mort. L'effet recherché par ces traductions n'est pas de conduire le lecteur à imaginer des entités hybrides ou bizarres, mais de lui permettre d'appréhender la variation potentiellement infinie des entités et des éléments du cosmos marubo. Nous avons par ailleurs préféré des formes courtes ou abrégées, qui recréent l'économie poétique de l'original pano et rendent mieux sa compulsion de variation, à des traductions peut-être plus littérales (et beaucoup trop longues pour la construction rythmique des traductions).

21. Ne pas confondre "mingau » (motsá) et « infusion » (ene) : ce dernier terme renvoie à une décoction ou un thé de Brugmansia sp. ou d'autres plantes comme le tabac, l'ayahuasca ou le mata-pasto.

22. «Liane » désigne icil'ayahuasca consommée par les esprits.

23. Ils adjoignent à la torpeur de l'ayahuasca (oni sanĩ) leurs vents (we). Le terme sanĩ désigne plus précisément la vision trouble, la sensation d'ivresse et les flashes de lumière issus de l'ingestion de l'ayahuasca.

24. Ainsi classifié puisqu'il s'agit du pont traversé par les Rovonawavo (Peuple-Cassique). Chaque peuple possède son propre pont marqué, au cours de la narration, par ses propres classificateurs (« soleil », vari, « ara », kana, « jaguar », ino, « passereau », shane, entre autres).

25. Les « lames-eau » sont des éléments coupants situés au fond des fleuves sur lesquels je n'ai pas obtenu plus d'informations.

26. «Doubles solitaires » (mão vaká) : il s'agit de l'un des (divers) composants de la personne qui survit, seul, après sa désagrégation. Une traduction alternative serait « doubles orphelins » (vaká mãoa).

27. Noa voro wetsã, littéralement « autre colline du fleuve », est une métaphore pour la TerreFleuve (Noa Mai), terre du grand fleuve situé à l'aval (le Solimões, Noa).

28. Les Iskonawavo forment l'un des peuples ancestraux qui participèrent au voyage décrit dans le wenia saiti. Les autres étaient le Peuple-Soleil (Varinawavo), le Peuple-Ara (Kananawavo), le PeupleJaguar (Inonawavo) et le Peuple-Passereau (Shanenawavo). On observera que, selon le système de classification marubo, rovo et isko sont deux termes se référant à des espèces distinctes de cassiques (de la famille des Icteridae, Psarocolius spp.).

29. Dans les cosmologies amérindiennes, le «temps mythique» n'appartient pas à un passé inaccessible, mais constitue un champ de références virtuel parallèle à «l'actuel » (Viveiros de Castro 2002).

30. «Passereau » se réfère de manière générique à l'oiseau évêque bleu (Cyanocompsa cyanea).

31. Voir Viveiros de Castro (2002) pour les Yawalapiti ; Deshayes et Keifenheim (1994) pour les Kashinawa.

32. Pour une analyse plus approfondie de cet aspect, voir Cesarino (2008).

33. Sur le parallélisme dans les arts de la parole amérindiens, voir Monod Becquelin (1986), Severi (2007), Cesarino (2006) et Franchetto (2003).

34. L'étude des chants saiti par l'ethnomusicologue Werlang (2001) confirme ce point en ce qui concerne la relation entre structures musicales et compositions de formules. Il est possible que la musique constitue de fait le point de convergence des chaînes intersémiotiques et des codifications des formules, comme le suggère Menezes Bastos (2007). 
35. Traduction d'un chant entonné par Tekãpapa à ma demande. Cette version est très proche de celles qui ont été chantées durant la cérémonie.

36. En plus de la ressemblance visuelle entre le chemin sinueux et le corps d'un serpent, ce dernier fut, encore une fois, utilisé dans les premiers temps par le Peuple-Esprit des Arbres (Tama Yove Nawavo) pour construire le chemin. D'où l'usage de ces images. Pour plus de détails, voir Cesarino (2008).

37. «Os de tapir » est une métaphore du Pont-Anaconda sur lequel le défunt peut glisser : il tombe alors dans le Fleuve-Mort.

38. La carapace de la tortue-mort, disent les chamanes, n'est pas faite, en réalité, de l'écorce de l'arbre-mort, mais seulement de son cuir (qui recouvre les os). L'allusion à l'écorce de l'arbre est, une fois encore, poétique ou métaphorique.

39. Tortue-mort inflige la « fatigue » (pini akaya) aux défunts qui l'observent, coupant les tendons de leurs chevilles. Elle est allongée sur une feuille de taioba et sa carapace est faite de l'écorce de l'arbre-mort.

40. «Souche d'arbre-mort » est une métaphore pour les collines dans lesquelles vivent les peuples du Chemin-Mort.

41. Assis en ce lieu, le défunt va se transformer en une termitière (nakashe) dont seule la tête émerge. Le singe-mort (singe atèle) considère comme sa colline (où sont construits malocas et villages) les souches des arbres.

42. L'odeur puissante du sang provoque la fatigue, elle épuise l'« âme », le « principe vital » (nokẽ chinã yomekaya).

43. L'étang de sang-mort fut formé à partir d'un morceau de serpent, laissé jadis sur le chemin par les esprits démiurges.

44. Il s'agit d'un puissant vent qui permet au défunt de passer rapidement, comme un avion : vents d'arbre-soleil, d'arbre-passereau, d'arbre-mort.

45. Référence aux anciens parents décédés (shenirasĩ).

46. Les chanteurs maubo peuvent substituer les formules vei tama voro et vei tama teke, « tronc d'arbre-mort », l'une à l'autre. Elles sont traduites de la même façon. Voro et teke désignent un morceau, un tronc ou une souche d'arbre (tama voro, tama teke).

47. C'est pourquoi la formule « queue d'ara-mort » (vei shawã ina) sera utilisée, dans d'autres arts de la parole marubo, comme une métaphore désignant les flammes du foyer (txi e).

48. Ces considérations sont développées dans un autre article consacré aux relations entre dessins et arts verbaux chez les Marubo (Cesarino 2010a).

49. De haut en bas : le monde du Ciel-Mort (Vei Mai Shavaya), le monde du Ciel-Passereau (Shane Nai Shavaya), le monde du Ciel-Éclair (Kaná Nai Shavaya), le monde du Ciel-Sang (Imi Nai Shavaya) et le monde du Ciel-Dessin (Kene Nai Shavaya).

50. Voir Taylor (2003) pour une étude de ces genres d'expression.

51. Un répertoire de ce genre a été étudié chez les Walbiri par Munn (1986). Ce type d'iconographie développe très clairement une série de relations systématiques au langage. Les critiques de Gell (1998) à propos de l'inadéquation de la terminologie linguistique pour l'étude des expressions visuelles ne s'appliquent donc pas ici.

52. À propos des phénomènes d'intersémioticité associés aux graphismes, voir Vidal (1992), Van Velthem (2003), Barcelos Neto (2001).

53. La personne marubo possède plusieurs doubles parmi lesquels trois sont particulièrement importants : le double de la poitrine ou de la pensée (chinã nató), le double du côté droit (mekiri vaká) et le double du côté gauche (mechmiri vaká). Il s'agit d'une triade de frères, du plus vieux (et plus savant) au plus jeune (et plus insensé). Les doubles maintiennent des relations avec le champ sociocosmique : ils sont « presque comme des esprits » (yovepase) et c'est ainsi qu'ils développent les capacités intellectuelles de la personne. Isko Osho est le nom du double-frère le plus vieux de Robson Venãpa et donc le principal responsable du savoir transmis par ce chamane.

54. Voir aussi Course (2009), Viveiros de Castro (1986), Taylor (1983) et Fausto (2001). 
55. Au cours d'une conférence donnée à l'Institut de Estudos Brasileiros de l'Université de São Paulo, cycle « Sentidos de Lévi-Strauss », 09 octobre 2008.

56. Voir Cesarino (2008, 2010a, 2010b) pour plus de développements sur ce point et d'une manière générale sur le mode de pensée chamanique marubo en relation avec les problèmes de la mémoire, de la transmission, de la création et de l'autorité.

\section{RÉFÉRENCES CITÉES}

Almeida Mauro William Barbosa de

2008 "A fórmula canônica do mito », in Ruben Caixeta de Queiroz et Renarde Freire Nobre (éd.), Lévi-Strauss: leituras brasileiras, Belo Horizonte, Editora UFMG, Belo Horizonte, pp. 147-183.

Barcelos Neto Aristóteles

2001 «O universo visual dos xamãs wauja », Journal de la Société des Américanistes, 87, pp. 131-160.

Cesarino Pedro

2003 Palavras torcidas: metáfora e personificação nos cantos xamanísticos ameríndios, dissertação de mestrado em Antropologia Social, Universidade Federal do Rio de Janeiro, Museu Nacional, Rio de Janeiro.

2006 «De duplos e estereoscópios : paralelismo e personificação nos cantos xamanísticos ameríndios », Mana, 12 (1), pp. 105-134.

2008 Oniska. A poética do mundo e da morte entre os Marubo da Amazônia ocidental, tese de doutorado em Antropologia Social, Universidade Federal do Rio de Janeiro, Museu Nacional, Rio de Janeiro.

2010a «Cartografias do cosmos: imagem, palavra e conhecimento entre os Marubo » [manuscrit].

2010b «Donos e duplos : relações de autoria e propriedade entre os Marubo », Revista de Antropologia, 53 (1), São Paulo pp. 147-199.

Course Magnus

2009 "Why Mapuche sing», Journal of the Royal Anthropological Institute, 15 (2), pp. 295-313.

DÉLÉAGE Pierre

2006 Le chamanisme sharanahua (Enquête sur l'apprentissage et l'épistémologie d'un rituel), thèse de doctorat, École des Hautes Études en Sciences Sociales, Paris.

2007 «Les répertoires graphiques amazoniens », Journal de la Société des Américanistes, 93 (1), pp. 97-126.

2011 «Les pictographies narratives amérindiennes », in Christian Jacob (éd.), Les lieux de savoir. 2. Les mains de l'intellect, Albin Michel, Paris, pp. 744-764.

Deleuze Gilles et Félix Guattari

1991 Qu'est-ce que la philosophie? Éditions de Minuit, Paris.

Deshayes Patrick et Barbara KeIFENHEIM

1994 Penser l'Autre chez les Huni Kuin de l'Amazonie, L'Harmattan, Paris. 
Fausto Carlos

2001 Inimigos Fiéis, EDUSP, São Paulo.

FRANCHETTO Bruna

2003 «L'autre du même : parallélisme et grammaire dans l'art verbal des récits kuikuro (Caribe du Haut Xingu, Brésil) », Amérindia, 28, pp. 213-248.

GELL Alfred

1998 Art and agency, Clarendon Press, Oxford.

GuIMARãEs Daniel

2002 De que se faz um Caminho - tradução e leitura de cantos kaxinawá, dissertação de mestrado, Universidade Federal Fluminense, Niterói.

HugH-JoNES Stephen

1994 "Shamans, prophets, priests and pastors», in Caroline Humphrey et Nicholas Thomas (éd.), Shamanism, history and the state, University of Michigan Press, Ann Arbor, pp. 32-75.

LAGRou Elsje

1998 Caminhos, duplos e corpos, tese de doutorado, Universidade de São Paulo, São Paulo.

LÉvi-STRauss Claude

1964 Mythologiques. I. Le cru et le cuit, Plon, Paris.

LORD Albert

1981 The singer of tales, Atheneum, New York.

MANigLIER Patrice

2008 «La condition symbolique », Philosophie, 98, pp. 37-53.

Menezes Bastos Rafael José de

2007 «Música nas sociedades indígenas das terras baixas da América do Sul : estado da arte », Mana, 13 (2), pp. 293-317.

Monod BeCquelin Aurore

1986 «Le sang et le corps, ou le blanc et le noir ? Contribution à l'étude du parallélisme dans la tradition orale des maya », Journal de la Société des Américanistes, 72, pp. 7-31.

MONTAGNER Delvair

1996 A morada das almas, Museu Paraense Emílio Goeldi, Belém.

MunN Nancy

1986 Walbiri iconography, Chicago University Press, Chicago.

NaveIra Miguel Carid

2007 Yama yama: os sons da memória. Afetos e parentesco entre os Yaminahua, tese de doutorado, Universidade Federal de Santa Catarina, Florianópolis.

OAKDALE Suzanne

1996 The power of experience : agency and identity in Kayabi healing and political process in the Xingu indigenous park, $\mathrm{PhD}$ thesis, department of anthropology, University of Chicago, Chicago. 
2005 I foresee my life : the ritual performance of autobiography in an Amazonian community, University of Nebraska Press, Lincoln.

SEveri Carlo

1996 La memoria ritual, Ediciones Abya-Yala, Quito.

2007 Le principe de la chimère. Une anthropologie de la mémoire, Éditions rue d’Ulm/Musée du quai Branly, Paris.

SHERZER Joel

1990 Verbal art in San Blas, University of New Mexico Press, Albuquerque.

TAYLOR Anne Christine

1983 « Jivaroan magical songs : Achuar anent of connubial love », Amérindia, 8, pp. 87-127.

2003 «Les masques de la mémoire : essai sur la fonction des peintures corporelles jivaro », L'Homme, 165, pp. 223-248.

Tournon Jacques

1991 «Medicina y visiones : canto de un curandero shipibo-conibo, texto y contexto », Amérindia, 16, pp. 179-209.

TownsLey Graham

1993 "Song paths: the ways and means of Yaminawa shamanic knowledge », L'Homme, 126-128, pp. 449-468.

VALENZUEla Pilar

1998 "Luna-avispa” y "tigre-machaco" : compuestos semánticos en la taxonomia shipiba », in Zarina Estrada, Max Figueroa, Gerardo López et Andrés Costa (éd.), IV Encuentro internacional de linguística del Nordeste (memórias), tome 1, vol. 2, pp. 409-428.

2000 "Major categories in Shipibo ethnobiological taxonomy », Anthropological Linguistics, 42 (1), pp. 1-36.

VAN Velthem Lúcia Hussak

2003 O Belo é a fera, Assírio e Alvim/Museu Nacional de Etnologia, Lisboa.

VIDAL Lux (éd.)

1992 Grafismo indigena, Studio Nobel/Fapesp/ Edusp, São Paulo.

Viveiros de CASTro Eduardo

1986 Araweté, os deuses canibais, Zahar/Anpocs, Rio de Janeiro.

2002 A inconstância da alma selvagem, CosacNaify, São Paulo.

2006 " A floresta de cristal : notas sobre a ontologia dos espíritos amazônicos », Cadernos de Campo, 14-15, pp. 319-339.

2007 «Filiação intensiva e aliança demoníaca », Novos Estudos, 77, pp. 91-126.

2008 «Xamanismo transversal : Lévi-Strauss e a cosmopolítica amazônica », in Ruben Caixeta de Queiroz et Renarde Freire Nobre (éd.), Lévi-Strauss : leituras brasileiras, Editora UFMG, Belo Horizonte, pp. 79-125.

WERLANG Guilherme

2001 Emerging peoples : Marubo myth-chants, PhD thesis, University of Saint Andrews, Saint Andrews. 


\section{ANNEXES}

Les tableaux qui suivent donnent à voir une systématisation des correspondances entre les formules narratives, provenant des arts verbaux, et les signes graphiques utilisés par les chamanes dans leurs dessins. Le tableau 1 ne contient que les formules les plus fréquentes des chants, puisque la reproduction de toutes les séquences dans lesquelles elles sont insérées déborderait largement de ses limites (on trouvera d'autres exemples dans les fragments traduits dans cet article). Les tableaux 2, 3 et 4 sont composés de dessins entiers ou de détails qui font référenceà un répertoire sélectionné d'iconographiemarubo. L'objectif est de fournir des exemples de l'articulation entre les signes graphiques et les formules verbales.

\begin{tabular}{|c|c|c|c|c|c|c|}
\hline \multicolumn{7}{|c|}{ Correspondances entre formules narratives et signes graphiques } \\
\hline \multirow{2}{*}{$\begin{array}{l}\begin{array}{l}\text { Signes } \\
\text { graphiques }\end{array} \\
\text { cercle }\end{array}$} & \multirow{2}{*}{\begin{tabular}{|l|}
$\begin{array}{l}\text { Référence } \\
\text { correspondante }\end{array}$ \\
$\begin{array}{l}\text { colline } \\
\text { trou } \\
\text { nectar } \\
\text { villages }\end{array}$ \\
\end{tabular}} & \multirow{2}{*}{\begin{tabular}{|l|} 
Variation possible \\
a) lieu de \\
surgissement \\
b) lieu d'installation \\
\end{tabular}} & \multicolumn{2}{|c|}{ Moments correspondants } & \multicolumn{2}{|c|}{$\begin{array}{l}\text { Formules narratives } \\
\text { correspondantes (quelques } \\
\text { exemples) }\end{array}$} \\
\hline & & & $\begin{array}{l}\text { awẽ shovia, } \\
\text { awẽ wenía, } \\
\text { awẽ tsaoa }\end{array}$ & \begin{tabular}{|l|} 
sa formation \\
son \\
surgissement \\
son \\
installation \\
\end{tabular} & $\begin{array}{l}\text { X oni nãko I } \\
\text { nãko osõatõsho }\end{array}$ & $\begin{array}{l}\text { «nectar } \\
\mathrm{XY} / \mathrm{du} \\
\text { nectar } \\
\text { émerge }{ }^{\mathrm{I}}\end{array}$ \\
\hline trajet & $\begin{array}{l}\text { chemins } \\
\text { (terrestres, } \\
\text { fluviaux, } \\
\text { célestes) }\end{array}$ & $\begin{array}{l}\text { a) surgissement } \\
\text { b) migration }\end{array}$ & awẽ vai & son chemin & $\begin{array}{l}\text { owa } x \text { shavaya I } \\
\text { ivaini voita }\end{array}$ & \begin{tabular}{|l} 
« et au \\
village x \\
/ au village \\
ils s'en \\
vont »
\end{tabular} \\
\hline maloca & $\begin{array}{l}\text { villages } \\
\text { (toponyme) }\end{array}$ & $\begin{array}{l}\text { lieu d'installation } \\
\text { (maloca, village) }\end{array}$ & awẽ tsaoa & $\begin{array}{l}\text { son lieu } \\
\text { d'installa- } \\
\text { tion }\end{array}$ & $\begin{array}{l}\text { x vake nawavo / } \\
\text { anõ vesokãia }\end{array}$ & $\begin{array}{l}\text { "fils du } \\
\text { peuples x / } \\
\text { son lieu } \\
\text { d'éveil " "I }\end{array}$ \\
\hline $\begin{array}{l}\text { souche } \\
\text { d'arbre }\end{array}$ & $\begin{array}{l}\text { villages } \\
\text { (toponyme) }\end{array}$ & lieu d'installation & awẽ tsaoa & $\begin{array}{l}\text { son lieu } \\
\text { d'installa- } \\
\text { tion }\end{array}$ & \begin{tabular}{|l|}
$x$ voro \\
masotanairi I \\
nioi kaoi
\end{tabular} & $\begin{array}{l}\text { " en haut } \\
\text { du tronc } \mathrm{x} \\
/ \text { là ils vont } \\
\text { vivre " }{ }^{\mathrm{II}}\end{array}$ \\
\hline humanoïde & $\begin{array}{l}\text { personne } \\
\text { (anthroponyme) }\end{array}$ & $\begin{array}{l}\text { a) individu } \\
\text { b) collectif }\end{array}$ & $\begin{array}{l}\text { awẽ ane, } \\
\text { awẽ yanika }\end{array}$ & $\begin{array}{l}\text { son nom, } \\
\text { son aliment } \\
\text { (et autres } \\
\text { habitudes) }\end{array}$ & $\begin{array}{l}\text { varĩ vake } \\
\text { nawavo } \\
\text { shanẽ vake } \\
\text { nawavo } \\
\text { etc. }\end{array}$ & $\begin{array}{l}\text { fils du } \\
\text { peuple- } \\
\text { soleil, } \\
\text { fils du } \\
\text { peuple- } \\
\text { passereau, } \\
\text { etc. }\end{array}$ \\
\hline arbre & $\begin{array}{l}\text { personne } \\
\text { (anthroponyme) }\end{array}$ & $\begin{array}{l}\text { a) individu } \\
\text { b) collectif }\end{array}$ & awẽ shovia & sa formation & $\begin{array}{l}\text { x tama nãko I } \\
\text { nãko osõatõsho }\end{array}$ & $\begin{array}{l}\text { "nectar de } \\
\text { l'arbre } \mathrm{X} / \\
\text { du nectar } \\
\text { émerge " }{ }^{\mathrm{IV}}\end{array}$ \\
\hline
\end{tabular}

TABLEAU I

I. Référence à un des modes possibles de surgissement des doubles et des esprits : par l'action du principe transformationnel nãko, parfois utilisé comme une métaphore pour désigner le sperme (ere) d'un peuple-esprit donné. Nãko fait aussi référence à la sève sucrée de certains arbres ou encore à un hyper-aliment des esprits yovevo ayant la forme d'une pomme. Toutefois la formule en question est la plupart du temps utilisée dans son sens premier, celui de principe transformationnel. « $\mathrm{XY}$ » fait référence aux variations possibles de la formule : $\mathrm{X}$ aux classificateurs (comme « ara », « soleil », «passereau », entre autres) et Y aux éléments dont dérive le « nectar » (comme l'ayahuasca, l'arbre ou la terre). Vari mai nãkolnãko osõatõsho, « du nectar de l'arbre-soleil/du nectar ils surgissent »; Shane oni nãko/nãko osõatõsho, « du nectar de l'ayahuasca-passereau/du nectar ils surgissent », entre autres.

II. « Lieu d'éveil » est une métaphore désignant les malocas et les villages.

III. « En haut du tronc-X » est une autre métaphore désignant villages ou malocas ; le $\mathrm{X}$ indique la place où doit être situé le classificateur.

IV. Voir la note 52. Dans ce cas, "arbre » est une métaphore visuelle pour les personnes (et leurs principes transformationnels respectifs). 


\begin{tabular}{|l|l|l|l|l|l|}
\hline \multicolumn{7}{|c|}{ Répertoire de signes graphiques } \\
\hline humanoïde & maloca & trajet & souche d'arbre & cercle & arbre \\
\hline & & & & & \\
\hline
\end{tabular}

TABLEAU 2

\begin{tabular}{|c|c|c|}
\hline \multicolumn{3}{|c|}{ Signes composés (diachroniques, narration) } \\
\hline Sens de la narration & Gauche $>$ droite & \\
\hline $\begin{array}{l}\text { Séquences de la } \\
\text { narration }\end{array}$ & Arbre $>$ cercle $>$ chemin $>$ souche d'arbre $>$ humanoïde & \\
\hline Explication & $\begin{array}{l}\text { À partir du nectar (nãko), surgissement du maître des } \\
\text { animaux (shane minshô) du peuple passereau } \\
\text { (Shanenawavo), qui suit son chemin jusqu’à son village } \\
\text { (indiqué par le signe «tronc »). } \\
\text { La séquence graphique traduit précisément les formules } \\
\text { verbales suivantes : « surgissant du nectar de mata-pasto- } \\
\text { passereau / dans le tronc de mata-pasto-passereau / là ils } \\
\text { sont allés vivre» (shane kapi iãkõsh wenía / shane kapi } \\
\text { voro masotanáiri / nioi kaoi)!. }\end{array}$ & \\
\hline Sens de la narration & Droite > gauche & \\
\hline $\begin{array}{l}\text { Séquences de la } \\
\text { narration }\end{array}$ & $\begin{array}{l}\text { Maloca }>\text { cercle }>\text { chemin }>[\text { cercle }[\text { humanoïde }]]> \\
\text { chemin }>\text { [cercle }[\text { humanoïde] }>\infty\end{array}$ & \\
\hline Explication & $\begin{array}{l}\text { Début du chemin-esprit (yove vai), qui conduit le chamane } \\
\text { aux villages des esprits du monde arboré (tama shavá). } \\
\text { Chaque cercle relié par des traits indique le village (shavá) } \\
\text { d'un peuple-esprit représenté par le signe « humanoïde » } \\
\text { inséré au milieu du cercle. La séquence de villages n'a pas } \\
\text { de fin précise. }\end{array}$ & \\
\hline Sens de la narration & Bas $>$ haut & \\
\hline $\begin{array}{l}\text { Séquences de la } \\
\text { narration }\end{array}$ & Cercle $>$ trajet $>$ maloca/ humanoïde & \\
\hline Explication & $\begin{array}{l}\text { Après le surgissement dans une spirale de « vent de terre- } \\
\text { brume », les esprits démiurges Kana Voã rejoignent leurs } \\
\text { malocas. Kana Voã est à gauche ; son neveu, Roẽ Iso, est } \\
\text { à droite. Le dessin traduit les premiers vers du chant saiti } \\
\text { «formation de la terre-brume » (kỗ mai vana). }\end{array}$ & \\
\hline
\end{tabular}

TABLEAU 3

I. « Tronc de mata-pasto » est ici une métaphore pour les malocas et villages. 


\begin{tabular}{|c|c|c|}
\hline \multicolumn{3}{|c|}{ Signes composés (synchroniques, énumération) } \\
\hline Composition & Humanoïde + maloca & \\
\hline Explication & $\begin{array}{l}\text { Esprits-chefs du monde du ciel-passereau (shane naí } \\
\text { shavaya) devant sa maloca. }\end{array}$ & $13=4$ \\
\hline Composition & Humanoïde + maloca & \\
\hline Explication & $\begin{array}{l}\text { Esprit-chef du monde du ciel-brume (koi naí shavaya) } \\
\text { devant sa maloca }\end{array}$ & \\
\hline Composition & Cercle + humanoïde & \\
\hline Explication & $\begin{array}{l}\text { Collectif d'esprits démiurges Kana Voã dans son lieu de } \\
\text { surgissement, la spirale de « vent de terre-brume » (koĩ mai } \\
\text { we) }\end{array}$ & \\
\hline Composition & Humanoïde + trace & \\
\hline Explication & $\begin{array}{l}\text { Assis sur un pont-anaconda, Owa Mani (en grand) nomme } \\
\text { les ancêtres (en petit). Fragment du chant de surgissement } \\
\text { des ancêtres, le Wenía. }\end{array}$ & 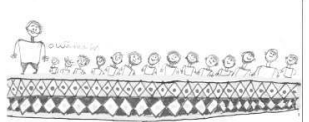 \\
\hline
\end{tabular}

TABLEAU 4 